\title{
Modeling and Control of UAV Bearing-Formations with Bilateral High-Level Steering
}

\author{
Antonio Franchi, Carlo Masone, Volker Grabe, Markus Ryll, Heinrich H. Bülthoff, and Paolo Robuffo Giordano
}

\begin{abstract}
In this paper we address the problem of controlling the motion of a group of UAVs bound to keep a formation defined in terms of only relative angles (i.e., a bearing-formation). This problem can naturally arise within the context of several multi-robot applications such as, e.g., exploration, coverage, and surveillance. First, we introduce and thoroughly analyze the concept and properties of bearing-formations, and provide a class of minimally linear sets of bearings sufficient to uniquely define such formations. We then propose a bearing-only formation controller requiring only bearing measurements, converging almost globally, and maintaining bounded inter-agent distances despite the lack of direct metric information.

The controller still leaves the possibility to impose group motions tangent to the current bearing-formation. These can be either autonomously chosen by the robots because of any additional task (e.g., exploration), or exploited by an assisting human co-operator. For this latter 'human-in-the-loop' case, we propose a multi-master/multi-slave bilateral shared control system providing the co-operator with some suitable force cues informative of the UAV performance. The proposed theoretical framework is extensively validated by means of simulations and experiments with quadrotor UAVs equipped with onboard cameras. Practical limitations, e.g., limited field-of-view, are also considered.
\end{abstract}

\section{INTRODUCTION}

Multi-robot systems have been largely studied in mobile robotics due to their resilience to single-point failures and adaptability to different scenarios. Some of the most promising applications are exploration, coverage, and surveillance, see, e.g., [1], [2], [3], [4]. Among the others, Unmanned Aerial Vehicles (UAVs) are probably the most suitable robotic platform for remote exploration of large and/or unstructured areas since they possess large adaptability and potential pervasiveness in many different scenarios. This is also attested by the growing number of funded projects focused on UAV applications, see, e.g., [5], [6], [7].

A primary requirement in multi-robot research is to devise motion controllers based on only relative measurements [8]. This way, the controller is independent of the knowledge of the robot absolute positions in space and, thus, does not require presence of global localization systems such as GPS or SLAM algorithms (see, e.g., [9]). In the same spirit,

A. Franchi, C. Masone, V. Grabe, M. Ryll, and P. Robuffo Giordano are with the Max Planck Institute for Biological Cybernetics, Spemannstraße 38, 72076 Tübingen, Germany \{antonio.franchi, carlo.masone, volker.grabe, markus.ryll, prg\}@tuebingen.mpg.de.

H. H. Bülthoff is with the Max Planck Institute for Biological Cybernetics, Spemannstraße 38, 72076 Tübingen, Germany, and with the Department of Brain and Cognitive Engineering, Korea University, Anam-dong, Seongbukgu, Seoul, 136-713 Korea hhb@tuebingen.mpg.de. substantial research efforts have been devoted to the development of solutions based on standard, light-weight, lowenergy, and cheap sensors (like onboard cameras), rather than active and energetically-expensive sensing devices (like, e.g., laser/structured-light range-sensors) [3]. When mapping/surveillance tasks are based on relative measurements obtained by cameras, a parallel objective for the group of robots is the maintenance of a 3D formation that optimizes some suitable 'visually-motivated' performance criterion. For example, in the context of environmental coverage [3], one can minimize the overlap of the camera field-of-views (FOVs) or maximize the focus on some areas of interest. Similarly, in visualbased cooperative map building, the robot 3D formations can be carefully designed so as to facilitate the acquisition of those relative measurements strictly needed for solving the mutual localization problem [10] - a necessary prerequisite for accurate merging of the individual maps. Since, regardless of the particular application, cameras eventually provide as a direct measurement only a bearing (angular) information, one then naturally faces the fundamental and underlying problem of realizing and keeping a desired UAV 3D formation defined in terms of sole relative (inter-robot) bearings (i.e., a bearingformation).

Furthermore, in real-world scenarios like search and rescue missions or exploration of highly dynamic human-populated environments, multi-robot systems seldom operate in full autonomy. In most cases, the cognitive skills of a human cooperator are still needed, either because of existing regulations requiring human presence/supervision, or because of the inherent complexity and unpredictability of the surrounding environment that exceeds the capabilities of nowadays robots. In fact, it is worth noting that in rescue operations the human-to-robot ratio is typically larger than 1 , thus indicating the average need of more than one human for operating a single robot [11]. When the human-robot interaction is made 'bilateral', i.e., suitable cues (such as haptic) are provided to the human co-operator in order to let her/him 'feel' the remote robot(s) and site [12], it has been proven that the human situational awareness and skill in task execution significantly increase, see, e.g., [13], [14].

Taking into account these considerations, in this paper we then pursue a twofold goal: on one side, and as main contribution, we propose a formation controller based on only relative bearing measurements for allowing a group of UAVs to reach and keep a desired 3D bearing-formation. We do not focus on a particular shape for the desired formation but assume it being specified by any 'external' task, for example optimal coverage, mapping, or cooperative transportation. Fur- 
thermore, and as second contribution, we allow the remaining degrees of freedom (dofs) of the formation to be externally controlled while fulfilling the relative bearing constraints. This possibility can be exploited by any higher-level navigation algorithm for, e.g., exploration purposes, or, as in the case considered in this paper, by human co-operators in charge of steering the whole group by acting on force-feedback interfaces. A suitable bilateral controller is then designed to provide force cues informative of how well the UAVs, as a group, are executing the humans' intended motion plan, i.e., so as to improve their situational awareness and overall quality of the human-robot cooperation during the robot steering.

The paper is organized as follows: Sec. I-A reviews the existing literature and better illustrates the main contributions of our work. Section II introduces the multi-UAV modeling assumptions, and Sec. III formally analyzes the main properties of 3D bearing-formations. The design of the bearing formation controller is detailed in Sec. IV, while Sec. V focuses on the application of the high-level steering to the case of bilateral interfaces. Finally, Secs. VII and VIII present the testbed and the obtained experimental results, and future directions are discussed in Sec. IX.

\section{A. Related Works and Contribution}

The previous works related to the topics of this paper can be roughly grouped in the following two categories:

1) Bearing/Vision-based Formation control: in the literature, the use of bearing measurements has been mainly explored for groups of non-holonomic ground (2D) robots with a special attention to leader-follower configurations.

In [15] a leader-follower control based on input-output feedback linearization is proposed. Every robot can control bearing and distance from another robot, or a combination of them from two other robots, and obstacle points. The quantities needed for the controller are either retrieved by an omnidirectional camera or estimated via EKF. [16] present a visual-servoing approach exploiting motion segmentation from panoramic images to estimate position and velocity of the leaders. In [17] distance estimation from the leader using bearing plus acceleration measurements is achieved using an EKF and a neural network. In [18] a leader-follower approach based on feedback linearization is proposed. The relative pose (bearing, distance and orientation) is estimated directly from the image using fiducial markers and a high gain non-linear observer. In [19] parallel and circular flocking formations (i.e., with constant non-zero speed) are obtained using the bearing angle, optical flow and time to collision.

Even though all the aforementioned approaches do not assume availability of distance measurements, they also aim at concurrently regulating all the robot inter-distances. This is typically achieved by fusing bearing-only measurements with additional metric information such as acceleration, velocity, or known size of objects. Therefore the fulfillment of suitable observability conditions must be taken into account as, e.g., shown in [20], as well as the maintenance of some sort of persistency of excitation condition during the motion. This can be obtained by either requiring the presence of a leader in charge of constantly "pulling" the formation, or by perturbing the motion of the robots with sinusoidal-like inputs.

However, such a persistent-excitation behavior is not needed by our formation controller, which neither uses nor regulates the inter-distances between the robots (while albeit ensuring their boundedness). The lack of a metric scale could be overcome, in some limited situations, by fusing visual information with onboard accelerometer readings as done in [21], [22]. However, for the sake of generality, in this paper we simply chose to discard any depth/scale estimation step, and to rather delegate to the high-level planner (a human co-operator in our case) the role of regulating the expansion/contraction rate of the UAV group. Nevertheless, the machinery presented in this paper can be easily extended to take advantage of a possibly available metric scale.

2) High-level steering of multiple mobile robots: any approach involving a group of robots tracking a collective reference trajectory can be loosely considered as non-bilateral (unilateral) steering of multiple mobile robots. For instance, in [23] a group of robots is made able to track given trajectories in the reduced space of some global quantities (e.g., the centroid of the formation), and conceptually similar problems are addressed in [24], [25] and references therein. Compared to our case, all these approaches: i) propose different solutions to multi-robot formation control, ii) do not focus on the specific case of bearing-only measurements, and iii) only consider the unilateral steering case.

While many papers in the past literature did consider bilateral teleoperation of a single mobile robot, only a few addressed the multiple mobile robot case. In [26] a passivity-based approach to bilaterally teleoperate a group of holonomic/non-holonomic ground robots is presented, and in [27] bilateral teleoperation of a group of UAVs is realized by directly coupling the position of the haptic device to the position of the formation centroid. This solution does not take into account the kinematic dissimilarity between the haptic device and the slave mobile robots (bounded vs. unbounded workspace), which, on the contrary, is explicitly considered in [28]. Here the authors present a UAV bilateral teleoperation scheme where the velocity of the group is controlled by the position of the haptic device, while still guaranteeing passivity (I/O stability) of the teleoperation system - a paradigm also followed by our present work. Along similar lines, in [29], [30], [31], [32], [33], [34] a different bilateral teleoperation control strategy is proposed with the emphasis on the possibility to allow autonomous split and rejoin decisions within the group in a passive/stable way. Nevertheless, all the aforementioned approaches are not bearing-only based but explicitly require knowledge of metric information.

Main contributions: Summarizing, the main contributions of our paper w.r.t. prior literature are the following:

1) we introduce and rigorously analyze the concept of $3 D$ bearing-formations, and describe the minimal sets of bearings needed to unequivocally define a bearingformation with linear cardinality in the number of robots. This allows to minimize the number of measurements needed to actually control the formation, thus improving the decentralization of the proposed scheme; 
2) we propose a $3 \mathrm{D}$ bearing-formation controller for UAVs based on only relative bearings. The controller is proven to be almost globally convergent, to stabilize the UAV inter-distances to a finite value despite the lack of metric measurements, and to not require any persistency of excitation condition to accomplish this task;

3) we formally characterize the generalized velocities of the remaining dofs of a UAV group constrained to maintain a desired bearing-formation. We allow a highlevel steering module to command these dofs. When this module is a human co-operator, we propose a bilateral teleoperation system delivering a direct feeling of how well the group of UAVs is executing the motion commands;

4) we validate the theoretical claims and proposed machinery by means of extensive human/hardware-in-the-loop simulations and real experiments employing a group of quadrotors with onboard cameras and two forcefeedback devices. We also consider practical issues such as limited field-of-view of the onboard cameras by exploiting a simple estimation scheme during the teleoperation task.

\section{PRELIMINARIES}

\section{A. UAV Model}

The multi-robot system is composed of $N$ UAVs modeled as rigid bodies in space, with $N \geq 3$. The inertial (world) frame and the body frame attached to the center of mass of the $i$-th UAV are denoted with $\mathcal{W}:\left\{O_{\mathcal{W}}, X_{\mathcal{W}}, Y_{\mathcal{W}}, Z_{\mathcal{W}}\right\}$ and $\mathcal{A}_{i}$ : $\left\{O_{\mathcal{A}_{i}}, X_{\mathcal{A}_{i}}, Y_{\mathcal{A}_{i}}, Z_{\mathcal{A}_{i}}\right\}$, with $O_{*}$ and $X_{*}, Y_{*}, Z_{*}$ denoting the origins and the orthogonal axes of the frames, respectively. The configuration of the $i$-th UAV is represented by the position ${ }^{\mathcal{W}} \boldsymbol{p}_{\mathcal{A}_{i}} \in \mathbb{R}^{3}$ of $O_{\mathcal{A}_{i}}$ in $\mathcal{W}$ and by the rotation matrix ${ }^{\mathcal{W}} R_{\mathcal{A}_{i}} \in$ $S O(3)$ from $\mathcal{W}$ to $\mathcal{A}_{i}$. Rotations may also be represented in terms of the roll $\phi_{\mathcal{A}_{i}}$, pitch $\theta_{\mathcal{A}_{i}}$ and yaw $\psi_{\mathcal{A}_{i}}$ angles. In the following we shall use boldfaced symbols to denote vectors and normal weighted characters for scalars, as done, e.g., for ${ }^{\mathcal{W}} \boldsymbol{p}_{\mathcal{A}_{i}}$ and $\psi_{\mathcal{A}_{i}}$.

We assume that every UAV can locally measure the roll and pitch angles $\left(\phi_{\mathcal{A}_{i}}, \theta_{\mathcal{A}_{i}}\right)$, e.g., by exploiting onboard inertial measurement units (IMUs consisting of accelerometers and gyros) and standard estimation algorithms such as complementary filters [35]. However, the (absolute) yaw angle $\psi_{\mathcal{A}_{i}}$ cannot be recovered from standard IMUs, but one needs additional sensors, such as a compass, with typically a more limited reliability (e.g., compasses do not work well indoor or close to strong magnetic fields). Therefore, for the sake of generality, in this work we aim for an approach not strictly requiring the absolute yaw angle, but still able to make use of it when available.

Furthermore, we assume that the $i$-th UAV is able to measure the relative bearing pointing towards the center of mass of a $j$-th UAV and expressed in the body frame $\mathcal{A}_{i}$. This is formally defined as

$$
\boldsymbol{\beta}_{\mathcal{A}_{i} \mathcal{A}_{j}}={ }^{\mathcal{A}_{i}} R_{\mathcal{W}} \frac{{ }^{\mathcal{W}} \boldsymbol{p}_{\mathcal{A}_{j}}-{ }^{\mathcal{W}} \boldsymbol{p}_{\mathcal{A}_{i}}}{\left\|{ }^{\mathcal{W}} \boldsymbol{p}_{\mathcal{A}_{j}}-{ }^{\mathcal{W}} \boldsymbol{p}_{\mathcal{A}_{i}}\right\|} \in \mathbb{S}^{2}
$$

where, as usual, a subscript/superscript shift denotes the inverse/transpose of a rotation matrix. We stress that a direct measure of $\boldsymbol{\beta}_{\mathcal{A}_{i} \mathcal{A}_{j}}$ can be obtained by means of a calibrated camera mounted on the $i$-th UAV, thus freeing from the need of knowing the absolute positions ${ }^{\mathcal{W}} \boldsymbol{p}_{\mathcal{A}_{j}}$ and ${ }^{\mathcal{W}} \boldsymbol{p}_{\mathcal{A}_{i}}$ present in the definition (1).

Instead of assuming that the $i$-th UAV is able track any arbitrary trajectory $\left({ }^{\mathcal{W}} \boldsymbol{p}_{\mathcal{A}_{i}}(t),{ }^{\mathcal{W}} R_{\mathcal{A}_{i}}(t)\right) \in S E(3)$, we ask for the less demanding (but more feasible) requirement of a UAV able to track any smooth reference trajectory $\left(\boldsymbol{p}_{i}(t), \psi_{i}(t)\right) \in$ $\mathbb{R}^{3} \times \mathbb{S}^{1}$. A sufficient condition satisfying this assumption is that the UAV position and yaw angle $\left({ }^{\mathcal{W}} \boldsymbol{p}_{\mathcal{A}_{i}}, \psi_{\mathcal{A}_{i}}\right)$ are flat outputs [36], i.e, algebraically defining, with their derivatives, the state and control inputs of the UAV. Differential flatness is essentially equivalent to exact dynamic feedback linearizability with $\left({ }^{\mathcal{W}} \boldsymbol{p}_{\mathcal{A}_{i}}, \psi_{\mathcal{A}_{i}}\right)$ taken as linearizing outputs [37]. Helicopters and quadrotors are examples of differentially flat systems [38], [39].

\section{B. Agent Model}

In order to generate a feasible motion $\left(\boldsymbol{p}_{i}(t), \psi_{i}(t)\right)$ to be tracked by the $i$-th UAV, we consider a virtual kinematic system (henceforth called agent) whose inputs are a bodyframe linear velocity $\boldsymbol{u}_{i} \in \mathbb{R}^{3}$ and a yaw-rate $w_{i} \in \mathbb{R}$

$$
\left(\begin{array}{c}
\dot{\boldsymbol{p}}_{i} \\
\dot{\psi}_{i}
\end{array}\right)=\left(\begin{array}{cc}
R_{i} & \mathbf{0}_{3} \\
\mathbf{0}_{3}^{T} & 1
\end{array}\right)\left(\begin{array}{c}
\boldsymbol{u}_{i} \\
w_{i}
\end{array}\right),
$$

where $\mathbf{0}_{3}=\left(\begin{array}{lll}0 & 0 & 0\end{array}\right)^{T}$ and $R_{i}=R_{z}\left(\psi_{i}\right)$, the canonical rotation matrix about the $Z$ axis. The rotation matrix between the body frames of agents $i$ and $j$ is ${ }^{i} R_{j}={ }^{i} R R_{j}$, where as usual ${ }^{i} R=R_{i}^{T}$. We also define the $i$-th agent configuration as $\boldsymbol{q}_{i}=$ $\left(\boldsymbol{p}_{i}, \psi_{i}\right) \in \mathbb{R}^{3} \times \mathbb{S}^{1}$ and collect all of them in a configuration vector $\boldsymbol{q}=\left(\boldsymbol{q}_{1}, \ldots, \boldsymbol{q}_{N}\right) \in\left(\mathbb{R}^{3} \times \mathbb{S}^{1}\right)^{N}$.

Analogously to (1), we define the agent relative bearing between the $i$-th and $j$-th agent (seen from the body frame of the $i$-th agent) as

$$
\boldsymbol{\beta}_{i j}(\boldsymbol{q})=\boldsymbol{\beta}_{i j}\left(\boldsymbol{q}_{i}, \boldsymbol{p}_{j}\right)={ }^{i} R \boldsymbol{p}_{i j} / \delta_{i j} \in \mathbb{S}^{2},
$$

where $\boldsymbol{p}_{i j}=\boldsymbol{p}_{j}-\boldsymbol{p}_{i}$, and

$$
\delta_{i j}(\boldsymbol{q})=\delta_{i j}\left(\boldsymbol{p}_{i}, \boldsymbol{p}_{j}\right)=\left\|\boldsymbol{p}_{j}-\boldsymbol{p}_{i}\right\|
$$

is the inter-distance between agents $i$ and $j$. We will omit the dependence from $\boldsymbol{q}$ when not needed or clear from the context. A configuration $\boldsymbol{q}$ is said degenerate if the associated agent positions $\boldsymbol{p}_{1}, \ldots, \boldsymbol{p}_{N}$ are all aligned, i.e., $\boldsymbol{\beta}_{12}(\boldsymbol{q})=$ $\pm \boldsymbol{\beta}_{13}(\boldsymbol{q})=\ldots= \pm \boldsymbol{\beta}_{1 N}(\boldsymbol{q})$. The reason for this definition will be clear in the next section.

Evaluation of $\boldsymbol{\beta}_{i j}$ can be obtained from the measured roll, pitch, and UAV relative bearing (1), as

$$
\boldsymbol{\beta}_{i j} \simeq R_{y}\left(\theta_{\mathcal{A}_{i}}\right) R_{x}\left(\phi_{\mathcal{A}_{i}}\right) \boldsymbol{\beta}_{\mathcal{A}_{i} \mathcal{A}_{j}}
$$

where $R_{x}(\cdot), R_{y}(\cdot)$ are the canonical rotation matrixes about the $X$ and $Y$ axes, respectively. The approximation (5) holds as long as the discrepancy between the position of the $j$-th UAV in the frame of the $i$-th UAV, i.e., ${ }^{\mathcal{A}_{i}} R_{\mathcal{W}}\left({ }^{\mathcal{W}} \boldsymbol{p}_{\mathcal{A}_{j}}-{ }^{\mathcal{W}} \boldsymbol{p}_{\mathcal{A}_{i}}\right)$, and that of the associated virtual agents, i.e., ${ }^{i} R\left(\boldsymbol{p}_{j}-\boldsymbol{p}_{i}\right)$, keeps small enough. This has been confirmed by the extensive set of simulations and experimental results reported in Sec. VIII. 


\section{MODELING OF BEARING-FORMATIONS}

\section{A. Useful properties of relative bearings}

The proofs of the following properties are reported in Appendix B. Denote with $[\boldsymbol{v}]_{\wedge} \in s o(3)$ the skew-symmetric matrix associated to a vector $\boldsymbol{v} \in \mathbb{R}^{3}$. We have:

Property 1. (Orientation) If $\boldsymbol{\beta}_{i j} \neq \pm\left(\begin{array}{lll}0 & 0 & 1\end{array}\right)^{T}$ then

$$
{ }^{i} R_{j}=R\left(\boldsymbol{\beta}_{i j}, \boldsymbol{\beta}_{j i}\right)=\exp \left(\arccos \left(c_{i j}\right)\left[\boldsymbol{v}_{i j}\right]_{\wedge}\right)
$$

where $\boldsymbol{v}_{i j}=-\boldsymbol{\beta}_{i j} \times \boldsymbol{\beta}_{j i}$ and $c_{i j}=\boldsymbol{\beta}_{i j} \cdot \boldsymbol{\beta}_{j i}$. Moreover if $\boldsymbol{\beta}_{i j}= \pm\left(\begin{array}{lll}0 & 0 & 1\end{array}\right)^{T}$ but $\boldsymbol{\beta}_{i k} \neq \pm\left(\begin{array}{lll}0 & 0 & 1\end{array}\right)^{T}$ then

$$
{ }^{i} R_{j}={ }^{i} R_{k}{ }^{k} R_{j}=R\left(\boldsymbol{\beta}_{i k}, \boldsymbol{\beta}_{k i}\right) R\left(\boldsymbol{\beta}_{k j}, \boldsymbol{\beta}_{j k}\right) .
$$

For any 3 agents $l, m$, and $n$, we define the scalar ratios among relative inter-distances

$$
\gamma_{l m n}=\frac{\delta_{l n}}{\delta_{l m}} \in \mathbb{R}^{+}, \quad \gamma_{m l n}=\frac{\delta_{m n}}{\delta_{m l}} \in \mathbb{R}^{+},
$$

assuming $\boldsymbol{p}_{l}$ and $\boldsymbol{p}_{m}$ are not coincident.

Property 2. (Triangulation) Consider 3 agents $l, m$, and $n$, s.t. $\boldsymbol{p}_{l}, \boldsymbol{p}_{m}$, and $\boldsymbol{p}_{n}$ are not aligned, i.e., $\boldsymbol{\beta}_{l m} \neq \pm \boldsymbol{\beta}_{l n}$. Then the following identities hold:

$$
\begin{aligned}
& \gamma_{l m n}=\Gamma\left(\boldsymbol{\beta}_{m n}, \boldsymbol{\beta}_{m l}, \boldsymbol{\beta}_{l n}\right) \\
& \gamma_{m l n}=\Gamma\left(\boldsymbol{\beta}_{l n}, \boldsymbol{\beta}_{l m}, \boldsymbol{\beta}_{m n}\right),
\end{aligned}
$$

where the function $\Gamma:\left(\mathbb{S}^{2}\right)^{3} \rightarrow \mathbb{R}^{+}$is defined as

$$
\Gamma\left(\boldsymbol{\beta}_{1}, \boldsymbol{\beta}_{2}, \boldsymbol{\beta}_{3}\right)=\frac{\left\|\boldsymbol{\beta}_{1} \times \boldsymbol{\beta}_{2}\right\|}{\left\|\boldsymbol{\beta}_{3} \times R\left(\boldsymbol{\beta}_{3}, \boldsymbol{\beta}_{2}\right) \boldsymbol{\beta}_{1}\right\|} .
$$

If $\boldsymbol{\beta}_{l m}= \pm \boldsymbol{\beta}_{l n}$ but $\boldsymbol{\beta}_{l m} \neq \pm \boldsymbol{\beta}_{l o}$, we can still obtain $\gamma_{l m n}$ as:

$$
\gamma_{l m n}=\frac{\delta_{l n}}{\delta_{l o}} \frac{\delta_{l o}}{\delta_{l m}}=\Gamma\left(\boldsymbol{\beta}_{o n}, \boldsymbol{\beta}_{o l}, \boldsymbol{\beta}_{l n}\right) \Gamma\left(\boldsymbol{\beta}_{m o}, \boldsymbol{\beta}_{m l}, \boldsymbol{\beta}_{l o}\right) .
$$

We also extend the definition of $\gamma_{l m n}$ to the singular cases $l=n$ and $m=n$ as

$$
\gamma_{l m l}=0, \quad \gamma_{l m m}=1 .
$$

Property 3. (Composition) Consider 3 agents $l, m, n$ then, if $\boldsymbol{\beta}_{l m} \neq \pm \boldsymbol{\beta}_{l n}$, the following identity holds:

$$
\boldsymbol{\beta}_{m n}={ }^{m} R_{l} \frac{\boldsymbol{\beta}_{l n} \gamma_{l m n}-\boldsymbol{\beta}_{l m}}{\left\|\boldsymbol{\beta}_{l n} \gamma_{l m n}-\boldsymbol{\beta}_{l m}\right\|} .
$$

\section{B. Definition of bearing-formations}

A formation of configurations, or just formation, is a pair $(\mathcal{G}, \boldsymbol{q})$ consisting of a generic graph $\mathcal{G}=(\mathcal{V}, \mathcal{E})$ - where $\mathcal{V}=\{1, \ldots N\}$ and $\mathcal{E} \subseteq \mathcal{T}=\{(i, j) \in \mathcal{V} \times \mathcal{V} \mid i \neq j\}-$ and a configuration vector $\boldsymbol{q}=\left(\boldsymbol{q}_{1}, \ldots, \boldsymbol{q}_{N}\right)$ that assigns to every node $i \in \mathcal{V}$ the configuration $\boldsymbol{q}_{i}=\left(\boldsymbol{p}_{i}, \psi_{i}\right) \in \boldsymbol{q}$. This kind of 'graph-plus-configuration' structure is also called framework or point-formation in the literature, see, e.g. [40], [41].

A formation $(\mathcal{G}, \boldsymbol{q})$ is degenerate if $\boldsymbol{q}$ is degenerate. Two formations $(\mathcal{G}, \boldsymbol{q})$ and $\left(\mathcal{G}, \boldsymbol{q}^{\prime}\right)$ with the same graph $\mathcal{G}$ but different configurations $\boldsymbol{q}$ and $\boldsymbol{q}^{\prime}$ are said edge-equivalent if

$$
\boldsymbol{\beta}_{i j}(\boldsymbol{q})=\boldsymbol{\beta}_{i j}\left(\boldsymbol{q}^{\prime}\right) \quad \forall(i, j) \in \mathcal{E},
$$

and similar if

$$
\boldsymbol{\beta}_{i j}(\boldsymbol{q})=\boldsymbol{\beta}_{i j}\left(\boldsymbol{q}^{\prime}\right) \quad \forall(i, j) \in \mathcal{T} .
$$

Finally, a formation $(\mathcal{G}, \boldsymbol{q})$ is rigid if all its edge-equivalent formations are also similar.

A formation of bearings, or simply a bearing-formation, is a pair $(\mathcal{G}, \boldsymbol{\alpha})$ made of a generic graph $\mathcal{G}=(\mathcal{V}, \mathcal{E})$ and a collection of $|\mathcal{E}|$ unit vectors $\boldsymbol{\alpha}=\left(\ldots, \boldsymbol{\alpha}_{i j}, \ldots\right) \in \mathbb{S}^{2}|\mathcal{E}|$ that assigns to every edge $(i, j) \in \mathcal{E}$ the unit vector $\boldsymbol{\alpha}_{i j} \in \boldsymbol{\alpha}$. ${ }^{1}$ A bearing-formation $(\mathcal{G}, \boldsymbol{\alpha})$ is said feasible if all the unit vectors contained in $\boldsymbol{\alpha}$ can simultaneously exist as actual relative bearings $\boldsymbol{\beta}_{i j}\left(\boldsymbol{q}^{\alpha}\right)$ for some configuration $\boldsymbol{q}^{\alpha}$, i.e., such that $\boldsymbol{\beta}_{i j}\left(\boldsymbol{q}^{\alpha}\right)=\boldsymbol{\alpha}_{i j} \forall(i, j) \in \mathcal{E}$. In this case, $\left(\mathcal{G}, \boldsymbol{q}^{\alpha}\right)$ is called a realization of $(\mathcal{G}, \boldsymbol{\alpha})$.

Within the class of feasible bearing-formations, we define rigid bearing-formations those having only rigid realizations, and degenerate bearing-formations those having at least a degenerate realization. This also implicitly defines the cases of (feasible) non-rigid and non-degenerate bearing-formations. Two rigid bearing-formations $(\mathcal{G}, \boldsymbol{\alpha})$ and $\left(\mathcal{G}^{\prime}, \boldsymbol{\alpha}^{\prime}\right)$ are said equivalent if (16) holds for any pair of realizations $\left(\mathcal{G}, \boldsymbol{q}^{\alpha}\right)$ and $\left(\mathcal{G}^{\prime}, \boldsymbol{q}^{\alpha \prime}\right)$ of $(\mathcal{G}, \boldsymbol{\alpha})$ and $\left(\mathcal{G}^{\prime}, \boldsymbol{\alpha}^{\prime}\right)$, respectively. Therefore, two equivalent rigid bearing-formations share the same set of realizations.

Lemma 1. (Parameterization of a bearing-formation) The equivalence class of all the realizations of a non-degenerate and rigid bearing-formation $(\mathcal{G}, \boldsymbol{\alpha})$ is the set $\left\{\left(\mathcal{G}, \boldsymbol{q}^{\alpha}\right) \mid \boldsymbol{q}^{\alpha} \in\right.$ $\mathcal{Q}\}$ where $\mathcal{Q}$ is a 5-dimensional manifold embedded in $\left(\mathbb{R}^{3} \times \mathbb{S}^{1}\right)^{N}$, and globally parameterized by the configuration $\boldsymbol{q}_{i}^{\alpha}$ of one agent (4 parameters) and the distance $\delta_{i j}\left(\boldsymbol{q}^{\alpha}\right)$ between two agents (1 parameter).

The proof of the Lemma is reported in Appendix B.

To summarize, a bearing-formation is rigid if it is feasible and implicitly defines all the relative bearings of its realizations, i.e., if it provides the maximum information obtainable using only relative bearings. Furthermore, if a rigid bearingformation is degenerate then no constraint exists among the inter-distances of its realizations, while, in case of nondegeneracy, the inter-distance ratios are strictly constrained, as shown in the proof of Lemma 1.

In a formation control context, e.g., when performing surveillance/coverage tasks, one typically aims at regulating all the relative bearings $\boldsymbol{\beta}_{i j}(\boldsymbol{q}),(i, j) \in \mathcal{T}$, to some desired values $\boldsymbol{\beta}_{i j}^{d}$, i.e., so that $\boldsymbol{\beta}(\boldsymbol{q}) \rightarrow \boldsymbol{\beta}^{d}=\left(\ldots, \boldsymbol{\beta}_{i j}^{d}, \ldots\right)_{(i, j) \in \mathcal{T}}$, where $\boldsymbol{\beta}(\boldsymbol{q})=\left(\ldots, \boldsymbol{\beta}_{i j}(\boldsymbol{q}), \ldots\right)_{(i, j) \in \mathcal{T}}$. Owing to the previous definitions, in particular rigidity, this requirement can be simplified as follows: if there exists a graph $\mathcal{G}=(\mathcal{V}, \mathcal{E})$ such that the (desired) bearing-formation $\left(\mathcal{G}, \boldsymbol{\beta}_{\mathcal{E}}^{d}\right)$ (where $\boldsymbol{\beta}_{\mathcal{E}}^{d}=$ $\left.\left(\ldots, \boldsymbol{\beta}_{i j}^{d} \ldots\right)_{(i, j) \in \mathcal{E}}\right)$ is rigid, then imposing $\boldsymbol{\beta}_{i j}(\boldsymbol{q}) \rightarrow \boldsymbol{\beta}_{i j}^{d}$, $\forall(i, j) \in \mathcal{E}$, will necessarily imply $\boldsymbol{\beta}_{i j}(\boldsymbol{q}) \rightarrow \boldsymbol{\beta}_{i j}^{d}, \forall(i, j) \in$ $\mathcal{T} \backslash \mathcal{E}$. Moreover, the desired bearing-formation $\left(\mathcal{G}, \boldsymbol{\beta}_{\mathcal{E}}^{d}\right)$ can be replaced by any equivalent bearing-formation $\left(\mathcal{G}^{\prime}, \boldsymbol{\beta}_{\mathcal{E}^{\prime}}^{d}\right)$

\footnotetext{
${ }^{1}$ Notice that here the term 'bearing' is used as a synonym of 'unit vector'. Moreover, this formal definition of a bearing-formation is conceptually decoupled from the relative bearings associated to a specific formation of agents $(\mathcal{G}, \boldsymbol{q})$ introduced before. Note also the 'duality' between the definitions of $(\mathcal{G}, \boldsymbol{q})$ and $(\mathcal{G}, \boldsymbol{\alpha})$.
} 


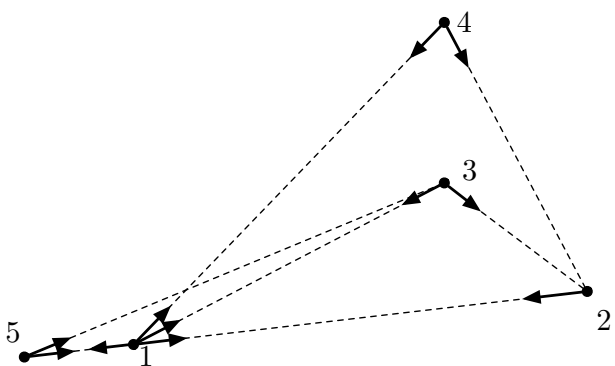

Fig. 1: Construction of the minimally-linear rigid bearing-formation described in Lemma 2 for the case of 5 agents. Agents 1, 2, and 5 are aligned, therefore $\mathcal{I}_{2}=\{3,4\}, \mathcal{I}_{3}=\{5\}$, and, as a consequence, $\hat{\mathcal{E}}=\{(1,2),(1,3),(1,4),(1,5),(2,1),(3,1),(4,1),(5,1)\}$ $\cup\{(3,2),(4,2)\} \cup\{(5,3)\}$. Note that $|\hat{\mathcal{E}}|=3 \cdot 5-4=11$ instead of $|\mathcal{T}|=(5-1) \cdot 5=20$ (the number of all possible relative bearings).

for realizing the same formation control goal. Therefore, in order to reduce the number of measured quantities and the overall computational load of the formation controller, a minimal number of controlled and measured relative bearings is desirable. This then raises the issue of minimality for rigid bearing-formations, i.e., of the minimal cardinality of $|\mathcal{E}|$ needed to define a rigid bearing-formation.

Although $\mathcal{E}$ can contain up to $|\mathcal{T}|=N(N-1)=$ $O\left(N^{2}\right)$ pairs, Lemma 1 shows that any rigid non-degenerate bearing-formation defines a 5 -dimensional manifold in a $4 N$-dimensional space. Therefore, only $4 N-5$ independent constraints are actually needed to determine a bearingformation. This fact motivates us to call a non-degenerate and rigid bearing-formation $(\mathcal{G}, \boldsymbol{\alpha})$ minimally-linear rigid if any $\left(\mathcal{G}^{\prime}, \boldsymbol{\alpha}^{\prime}\right)$ with $\mathcal{E}^{\prime} \subsetneq \mathcal{E}, \boldsymbol{\alpha}^{\prime} \subsetneq \boldsymbol{\alpha}$ is not rigid, and $|\mathcal{E}|=O(N)$. The following Lemma, whose proof is again in Appendix B, explicitly constructs an important class of minimally-linear rigid bearing-formations which will then be used by our formation controller.

Lemma 2 (A minimally-linear rigid bearing formation). Given a non-degenerate rigid bearing-formation $(\mathcal{G}, \boldsymbol{\alpha})$, consider any corresponding realization $\left(\mathcal{G}, \boldsymbol{q}^{\alpha}\right)$. Then there exists at least one agent relabeling such that $\boldsymbol{\beta}_{12}\left(\boldsymbol{q}^{\alpha}\right) \neq \pm \boldsymbol{\beta}_{13}\left(\boldsymbol{q}^{\alpha}\right) \neq$ $\pm(001)^{T}$. Consider any one of these relabelings and define the following sets $\mathcal{I}_{2}, \mathcal{I}_{3} \subset \mathcal{V}$ and $\hat{\mathcal{E}} \subsetneq \mathcal{T}$ :

$$
\begin{aligned}
\mathcal{I}_{2} & =\{3\} \cup\left\{j \in\{4, \ldots, N\} \mid \boldsymbol{\beta}_{1 j}\left(\boldsymbol{q}^{\alpha}\right) \neq \pm \boldsymbol{\beta}_{12}\left(\boldsymbol{q}^{\alpha}\right)\right\} \\
\mathcal{I}_{3} & =\{4, \ldots, N\} \backslash \mathcal{I}_{2} . \\
\hat{\mathcal{E}} & =\{(1, j),(j, 1)\}_{j=2}^{N} \cup\{(j, 2)\}_{j \in \mathcal{I}_{2}} \cup\{(j, 3)\}_{j \in \mathcal{I}_{3}} .
\end{aligned}
$$

Define also $\hat{\mathcal{G}}=(\mathcal{V}, \hat{\mathcal{E}})$ and $\boldsymbol{\beta}_{\hat{\mathcal{E}}}^{\alpha}=\left(\ldots, \boldsymbol{\beta}_{i j}\left(\boldsymbol{q}^{\alpha}\right), \ldots\right)_{(i, j) \in \hat{\mathcal{E}}}$. Then the bearing-formation $\left(\hat{\mathcal{G}}, \boldsymbol{\beta}_{\hat{\mathcal{E}}}^{\alpha}\right)$ is non-degenerate, minimally-linear rigid, and equivalent to $(\mathcal{G}, \boldsymbol{\alpha})$.

As an example, Fig. 1 represents the construction of a minimally-linear rigid set in the case of 5 agents where agents 1,2 and 5 are aligned.

\section{CONTROL OF BEARING-FORMATIONS}

As introduced in the previous section, we will denote with $\left(\mathcal{G}, \boldsymbol{\beta}_{\mathcal{E}}^{d}\right)$ the desired rigid and non-degenerate bearingformation specifying the formation-control goal, and with
$(\mathcal{G}, \boldsymbol{q})$ the formation (of configurations) consisting of the same graph $\mathcal{G}$ and of the current agent configuration $\boldsymbol{q}$. In this section we propose a formation controller that, starting from a generic $\boldsymbol{q}\left(t_{0}\right)$, brings and maintains $(\mathcal{G}, \boldsymbol{q}(t))$ within the class of realizations of $\left(\mathcal{G}, \boldsymbol{\beta}_{\mathcal{E}}^{d}\right)$ (the control objective) by using only a minimally linear number of relative-bearing measurements. In addition, we also complement this control action by deriving the 'orthogonal' set of virtual inputs able to steer $(\mathcal{G}, \boldsymbol{q}(t))$ inside the class of realizations of $\left(\mathcal{G}, \boldsymbol{\beta}_{\mathcal{E}}^{d}\right)$.

To this aim, we split the control inputs of the $i$-th agent (2) into two terms

$$
\left(\boldsymbol{u}_{i}, w_{i}\right)=\left(\boldsymbol{u}_{i}^{h}, w_{i}^{h}\right)+\left(\boldsymbol{u}_{i}^{f}, w_{i}^{f}\right) .
$$

The term $\left(\boldsymbol{u}_{i}^{h}, w_{i}^{h}\right)$, described in the following Sec. IV-A, will be exploited to vary $(\mathcal{G}, \boldsymbol{q}(t))$ while preserving the relative bearings. Therefore, if $\left(\mathcal{G}, \boldsymbol{q}\left(t_{0}\right)\right)$ is a realization of $\left(\mathcal{G}, \boldsymbol{\beta}_{\mathcal{E}}^{d}\right)$, the control inputs $\left(\boldsymbol{u}_{i}^{h}, w_{i}^{h}\right)$ will make $(\mathcal{G}, \boldsymbol{q}(t))$ to evolve within the class of realizations of $\left(\mathcal{G}, \boldsymbol{\beta}_{\mathcal{E}}^{d}\right)$. Recalling the Introduction, $\left(\boldsymbol{u}_{i}^{h}, w_{i}^{h}\right)$ represents the action of any 'external high-level planner' in charge of steering the collective motion of the UAV group. The term $\left(\boldsymbol{u}_{i}^{f}, w_{i}^{f}\right)$, enforcing convergence to the desired bearing formation $\left(\mathcal{G}, \boldsymbol{\beta}_{\mathcal{E}}^{d}\right)$, is illustrated in Sec. IV-B.

\section{A. High-level Steering Preserving the Relative Bearings}

Consider the bearing-formation $(\mathcal{K}, \boldsymbol{\beta}(\boldsymbol{q}))$ where $\mathcal{K}=(\mathcal{V}, \mathcal{T})$ (the complete graph) and, as usual, $\boldsymbol{q}$ is the current configurations of the agents. The class of realizations of $(\mathcal{K}, \boldsymbol{\beta}(\boldsymbol{q}))$, denoted with $\mathcal{F}_{\mathcal{K}}(\boldsymbol{q})$, is a 5-dimensional manifold, as shown in Lemma $1 .^{2}$

Denote with $\dot{\boldsymbol{q}}=\left(\dot{\boldsymbol{p}}_{1}^{T} \dot{\psi}_{1} \ldots \dot{\boldsymbol{p}}_{N}^{T} \dot{\psi}_{N}\right)^{T} \in \mathbb{R}^{4 N}$ the vector of generalized velocities of the agents. By plugging (20) into (2) it is clear that $\dot{\boldsymbol{q}}=\dot{\boldsymbol{q}}^{f}+\dot{\boldsymbol{q}}^{h}$, where $\dot{\boldsymbol{q}}^{f}$ and $\dot{\boldsymbol{q}}^{h}$ depend only on $\left(\boldsymbol{u}_{i}^{f}, w_{i}^{f}\right)$ and $\left(\boldsymbol{u}_{i}^{h}, w_{i}^{h}\right)$, respectively, with $i=1 \ldots N$. Our goal is to design $\left(\boldsymbol{u}_{i}^{h}, w_{i}^{h}\right)$ such that $\dot{\boldsymbol{q}}^{h}$ belongs to $T_{\boldsymbol{q}} \mathcal{F}_{\mathcal{K}}(\boldsymbol{q})$, the tangent space at $\boldsymbol{q}$ of $\mathcal{F}_{\mathcal{K}}(\boldsymbol{q})$. In this way the the highlevel commands $\left(\boldsymbol{u}_{i}^{h}, w_{i}^{h}\right)$ will not interfere with the formation control objective, since the current relative bearings $\boldsymbol{\beta}(\boldsymbol{q})$ will be preserved.

In order to provide an analytical expression for $T_{\boldsymbol{q}} \mathcal{F}_{\mathcal{K}}(\boldsymbol{q})$, we let $\hat{\boldsymbol{p}}_{i j}=\frac{\boldsymbol{p}_{i j}}{\delta_{i j}} \in \mathbb{S}^{2} \forall i \neq j$, and consider, by convention, $\hat{\boldsymbol{p}}_{i i}=\mathbf{0}_{3}, \forall i=1, \ldots, N$. We also define $S=\left[e_{3}\right]_{\wedge}, e_{3}=$ $\left(\begin{array}{lll}0 & 0 & 1\end{array}\right)^{T}$, denote with $\mathcal{R}(A)$ the range (or column) space of a matrix $A$, and with $I_{3} \in \mathbb{R}^{3 \times 3}$ the $3 \times 3$ identity matrix.

The following lemma is a preliminary step in view of the more general characterization of $T_{\boldsymbol{q}} \mathcal{F}_{\mathcal{K}}(\boldsymbol{q})$ given in Proposition 1 .

Lemma 3 (Bearing-invariant motions for 3 agents). If $N=3$ and $\boldsymbol{q}$ is non-degenerate, then $T_{\boldsymbol{q}} \mathcal{F}_{\mathcal{K}}(\boldsymbol{q})=\mathcal{R}\left(T_{3}\right)$, being

$$
T_{3}=\left[\begin{array}{cccccc}
I_{3} & \mathbf{0}_{3} & I_{3} & \mathbf{0}_{3} & I_{3} & \mathbf{0}_{3} \\
\mathbf{0}_{3}^{T} & 0 & \hat{\boldsymbol{p}}_{12}^{T} & 0 & \gamma_{123} \hat{p}_{13}^{T} & 0 \\
\mathbf{0}_{3}^{T} & 1 & -\left(S \boldsymbol{p}_{12}\right)^{T} & 1 & -\left(S \boldsymbol{p}_{13}\right)^{T} & 1
\end{array}\right]^{T} \in \mathbb{R}^{12 \times 5} .
$$

${ }^{2}$ The class $\mathcal{F}_{\mathcal{K}}(\boldsymbol{q})$ is the same class of realizations obtained by any other rigid bearing formation $\left(\mathcal{G}, \boldsymbol{\beta}_{\mathcal{E}}(\boldsymbol{q})\right)$, with $\mathcal{G}=(\mathcal{V}, \mathcal{E})$ and $\boldsymbol{\beta}_{\mathcal{E}}(\boldsymbol{q})=$ $\left(\ldots, \boldsymbol{\beta}_{i j}(\boldsymbol{q}), \ldots\right)_{(i, j) \in \mathcal{E}}$. Nevertheless, use of $\mathcal{K}$ instead of a generic $\mathcal{G}$ automatically guarantees rigidity of $(\mathcal{K}, \boldsymbol{\beta}(\boldsymbol{q}))$ for any configuration $\boldsymbol{q}$. 
The formal proof is reported in Appendix B. It is worth analyzing the geometrical meaning of the columns of $T_{3}$ to get an intuition on the actual agent motion they represent. To this end, let $\boldsymbol{t}_{i} \in \mathbb{R}^{12}$ be the $i$-th column of $T_{3}$.

A motion along $\left(t_{1} t_{2} t_{3}\right)$, i.e.,

$$
\dot{\boldsymbol{q}}=\left[\begin{array}{lll}
\boldsymbol{t}_{1} & \boldsymbol{t}_{2} & \boldsymbol{t}_{3}
\end{array}\right] \boldsymbol{\nu}=\left[\begin{array}{llllll}
I_{3} & \mathbf{0}_{3} & I_{3} & \mathbf{0}_{3} & I_{3} & \mathbf{0}_{3}
\end{array}\right]^{T} \boldsymbol{\nu}
$$

for some $\boldsymbol{\nu} \in \mathbb{R}^{3}$ represents a synchronized translation of the formation with velocity $\nu$.

A motion along $t_{4}$, i.e.,

$$
\dot{\boldsymbol{q}}=\boldsymbol{t}_{4} s=\left[\begin{array}{llllll}
\mathbf{0}_{3}^{T} & 0 & \hat{\boldsymbol{p}}_{12}^{T} & 0 & \gamma_{123} \hat{\boldsymbol{p}}_{13}^{T} & 0
\end{array}\right]^{T} s
$$

for some $s \in \mathbb{R}$ represents a synchronized expansion of the formation with expansion rate $s$. In particular, agent 1 does not move, agent 2 moves along the connecting line with 1 at speed $s$, and agent 3 moves along the connecting line with 1 at speed $\gamma_{123} \mathrm{~s}$, that is, exactly the speed needed to keep the relative bearings unchanged.

Finally, a motion along $t_{5}$, i.e.,

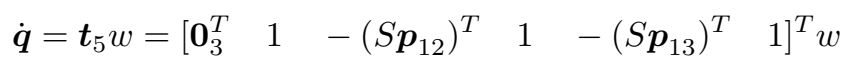

for some $w \in \mathbb{R}$ represents a synchronized rotation around agent 1 with rate $w$. Agent 1 rotates in place with an angular speed $\dot{\psi}_{1}=w$, agent 2 rotates with the same angular speed $\psi_{2}=w$ but is also translating around agent 1 with linear velocity $-\left(S \boldsymbol{p}_{12}\right) w$, and likewise for agent 3 . We also note that synchronized translations and expansions can be performed with the sole knowledge of relative bearings, while execution of a synchronized rotation necessarily requires an additional metric information (the magnitudes of $\boldsymbol{p}_{12}$ and $\boldsymbol{p}_{13}$ ).

Proposition 1 (Bearing-invariant motions for $N$ agents). Given a non-degenerate configuration $\boldsymbol{q}$, consider the sets $\mathcal{I}_{2}(\boldsymbol{q})$ and $\mathcal{I}_{3}(\boldsymbol{q})$ obtained by applying Lemma 2 to $(\mathcal{K}, \boldsymbol{\beta}(\boldsymbol{q}))$. Then, the set of motions keeping constant the relative bearings at $\boldsymbol{q}$ can always be expressed, possibly after an agent relabeling, as $T_{\boldsymbol{q}} \mathcal{F}_{\mathcal{K}}(\boldsymbol{q})=\mathcal{R}\left(T_{N}\right)$, where $T_{N}=$ $\left[\begin{array}{lll}T_{3}^{T} & T_{4}^{T} & T_{5}^{T}\end{array}\right]^{T} \in \mathbb{R}^{4 N \times 5}, T_{3}$ is defined in (21),

$$
\begin{aligned}
& T_{4}=\left[\begin{array}{cccc}
\cdots & I_{3} & \mathbf{0}_{3} & \cdots \\
\cdots & \gamma_{12 i} \hat{\boldsymbol{p}}_{1 i}^{T} & 0 & \cdots \\
\cdots & -\left(S \boldsymbol{p}_{1 i}\right)^{T} & 1 & \cdots
\end{array}\right]_{i \in \mathcal{I}_{2}(\boldsymbol{q}) \backslash\{3\}}^{T} \in \mathbb{R}^{4\left(\left|\mathcal{I}_{2}(\boldsymbol{q})\right|-1\right) \times 5} \\
& T_{5}=\left[\begin{array}{cccc}
\cdots & I_{3} & \mathbf{0}_{3} & \cdots \\
\cdots & \gamma_{13 i} \hat{\boldsymbol{p}}_{1 i}^{T} & 0 & \cdots \\
\cdots & -\left(S \boldsymbol{p}_{1 i}\right)^{T} & 1 & \cdots
\end{array}\right]_{i \in \mathcal{I}_{3}(\boldsymbol{q})}^{T} \in \mathbb{R}^{4\left|\mathcal{I}_{3}(\boldsymbol{q})\right| \times 5}
\end{aligned}
$$

Proof. Applying Lemma 2 to $(\mathcal{K}, \boldsymbol{\beta}(\boldsymbol{q}))$ yields the equivalent rigid and non-degenerate bearing-formation $\left(\hat{\mathcal{G}}, \boldsymbol{\beta}_{\hat{\mathcal{E}}}\right)$, where $\boldsymbol{\beta}_{\hat{\mathcal{E}}}=\left(\ldots, \boldsymbol{\beta}_{i j}(\boldsymbol{q}), \ldots\right)_{(i, j) \in \hat{\mathcal{E}}}$. Then the sought tangent space can be defined as the set of those velocities $\dot{\boldsymbol{q}}$ keeping all the bearings in $\boldsymbol{\beta}_{\hat{\mathcal{E}}}$ constant. Consider the sets composed by the relative bearings $\hat{\boldsymbol{\beta}}_{i}(\boldsymbol{q})=\left\{\boldsymbol{\beta}_{1 j}, \boldsymbol{\beta}_{j 1}, \boldsymbol{\beta}_{i 1}, \boldsymbol{\beta}_{1 i}, \boldsymbol{\beta}_{i j}, \boldsymbol{\beta}_{j i}\right\}$ where $j=2$, if $i \in \mathcal{I}_{2}(\boldsymbol{q})$ and $j=3$, if $i \in \mathcal{I}_{3}(\boldsymbol{q})$. It is clear that $\boldsymbol{\beta}_{\hat{\mathcal{E}}} \subset \cup_{i=3}^{N} \hat{\boldsymbol{\beta}}_{i}(\boldsymbol{q})$. Apply now Lemma 3 replacing agents 2 with $j$ and 3 with a generic agent $i \in\{3 \ldots N\}$, and consider the corresponding matrix $T_{i}(21)$ defined as

$$
T_{i}=\left[\begin{array}{cccccc}
I_{3} & \mathbf{0}_{3} & I_{3} & \mathbf{0}_{3} & I_{3} & \mathbf{0}_{3} \\
\mathbf{0}_{3}^{T} & 0 & \hat{\boldsymbol{p}}_{1 j}^{T} & 0 & \gamma_{1 j i} \hat{\boldsymbol{p}}_{1 i}^{T} & 0 \\
\mathbf{0}_{3}^{T} & 1 & -\left(S \boldsymbol{p}_{1 j}\right)^{T} & 1 & -\left(S \boldsymbol{p}_{1 i}\right)^{T} & 1
\end{array}\right]^{T} \in \mathbb{R}^{12 \times 5} .
$$

This matrix defines the motions of agents $1, j$, and $i$ which keep all the relative bearings in $\hat{\boldsymbol{\beta}}_{i}(\boldsymbol{q})$ constant. Note that the motions of agents 1 and $j$ are independent from the state of agent $i$. Therefore, in order to keep the bearings of all the subsets $\hat{\boldsymbol{\beta}}_{i}(\boldsymbol{q})$ constant, one can: i) choose the motion of agent 1 and 2 , then ii) select the motion of every agent $i \in \mathcal{I}_{2}(\boldsymbol{q})$ as per the last four rows of (24), with $j=2$, and finally iii) select the motion of every other agent $i \in \mathcal{I}_{3}(\boldsymbol{q})$ as per the last four rows of (24), with $j=3$. This procedure directly yields matrix $T_{N}$ and thus proves the Proposition.

Similarly to the previous case of 3 agents, the bearinginvariant motions represented by $T_{N}$ are of three kinds: a synchronized translation with velocity $\nu \in \mathbb{R}^{3}$, a synchronized expansion with rate $s \in \mathbb{R}$, and a synchronized rotation with speed $w \in \mathbb{R}$.

The following steps finally show how to design the control terms $\left(\boldsymbol{u}_{i}^{h}, w_{i}^{h}\right)$ in order to impose the aforementioned bearing-invariant motions to the UAV group. Without loss of generality, we will present our derivations assuming an external planner (as a human operator) controlling the group form the body-frame of agent 1 . From Prop. 1, this can be obtained by letting, $\forall i=1 \ldots N$,

$$
\begin{aligned}
\boldsymbol{u}_{i}^{h} & ={ }^{i} R_{1} \boldsymbol{\nu}-s \gamma_{12 i} \boldsymbol{\beta}_{i 1}+w \delta_{12} \gamma_{12 i} S \boldsymbol{\beta}_{i 1} \\
w_{i}^{h} & =w .
\end{aligned}
$$

where $\gamma_{12 i}$ is computed as $\gamma_{123} \gamma_{13 i}$ for those $i \in \mathcal{I}_{3}(\boldsymbol{q})$. It is possible to prove (the proof is omitted for brevity) that commands (25-26) will result in $\dot{\boldsymbol{p}}_{1}=R_{1} \nu, \dot{\psi}_{1}=w$, and $\dot{\delta}_{12}=s$ for agent 1 , and in exactly those coordinated motions preserving all the relative bearings, for all the remaining agents $i=2 \ldots N$,

We note that, again, control (25-26) is a function of only bearing measurements (using Properties 1 and 2) with the exception of the unique metric quantity $\delta_{12}$. This is in fact needed in (25) to correctly implement a synchronized group rotation. If $\delta_{12}$ is available through direct measurement or online estimation, then (25) can be exactly implemented. If the distance $\delta_{12}$ is not available then it can be replaced with an arbitrary initial guess $\hat{\delta}_{12}>0$, e.g., chosen by a high-level planner or a human co-operator. This choice will result in a non-perfect execution of the synchronized rotation command, which will pull the bearing-formation away from the desired one. On the other hand, the feedback action of the term $\left(\boldsymbol{u}_{i}^{f}, w_{i}^{f}\right)$ in (20) - see (27-29) - will keep these disturbances bounded by trying to achieve the desired formation, eventually keeping a (bounded) non-zero bearing error at steady-state. These intuitive considerations have been empirically proven by our simulations and experiments of Sec. VIII, while a formal characterization will be addressed in future works.

\section{B. Regulation to the Desired Bearing Formation}

Applying Lemma 2 with $\boldsymbol{\beta}_{\mathcal{E}}^{d}$ playing the role of $\boldsymbol{\alpha}$, the agents are able to compute a minimally-linear rigid bearingformation $\left(\hat{\mathcal{G}}, \boldsymbol{\beta}_{\hat{\mathcal{E}}}^{d}\right)$ equivalent to $\left(\mathcal{G}, \boldsymbol{\beta}_{\mathcal{E}}^{d}\right)$, thus sharing the same class of realizations. Agents 1,2 , and 3 , as per the definition of $\hat{\mathcal{E}}$, will be called beacon agents. We note that their role can be taken by any triplet whose positions in a realization of 
$\left(\mathcal{G}, \boldsymbol{\beta}_{\mathcal{E}}^{d}\right)$ (and then also of $\left(\hat{\mathcal{G}}, \boldsymbol{\beta}_{\mathcal{\mathcal { E }}}^{d}\right)$ ) are not collinear. Exploiting the desired bearings in $\boldsymbol{\beta}_{\hat{\mathcal{\varepsilon}}}^{d}$, the agents are also able to compute all the desired relative rotations ${ }^{1} R_{i}^{d}$ and desired distance ratios $\gamma_{12 i}^{d}$ for any $i=2 \ldots N$ by resorting to Properties 1 and 2, respectively. Then, we propose the following control law for the term $\left(\boldsymbol{u}_{i}^{f}, w_{i}^{f}\right)$ in $(20)$ :

$$
\begin{aligned}
\left(\begin{array}{l}
\boldsymbol{u}_{1}^{f} \\
w_{1}^{f}
\end{array}\right) & =\left(\begin{array}{c}
\mathbf{0} \\
0
\end{array}\right) \\
\left(\begin{array}{c}
\boldsymbol{u}_{2}^{f} \\
w_{2}^{f}
\end{array}\right) & =\left(\begin{array}{c}
K_{p}{ }^{2} R_{1}\left[\boldsymbol{\beta}_{12} \times\left(\boldsymbol{\beta}_{12}^{d} \times \boldsymbol{\beta}_{12}\right)\right] \\
K_{\omega}\left[{ }^{1} R_{2}^{d}{ }^{2} R_{1}-{ }^{1} R_{2}{ }^{2} R_{1}^{d}\right]_{\vee, 3}
\end{array}\right) \\
\left(\begin{array}{c}
\boldsymbol{u}_{i}^{f} \\
w_{i}^{f}
\end{array}\right) & =\left(\begin{array}{l}
K_{p}{ }^{i} R_{1}\left(\gamma_{12 i}^{d} \boldsymbol{\beta}_{1 i}^{d}-\gamma_{12 i} \boldsymbol{\beta}_{1 i}\right. \\
K_{\omega}\left[{ }^{1} R_{i}^{d}{ }^{i} R_{1}-{ }^{1} R_{i}{ }^{i} R_{1}^{d}\right] \\
\vee, 3
\end{array}\right)
\end{aligned}
$$

with $i=3, \ldots, N, K_{p}, K_{\omega}>0$ being positive gains, and $[A]_{\vee, 3}$ representing the third component of the vector associated to a skew-symmetric matrix $A$. We can then prove the following statement:

Proposition 2 (Formation Control). Given a desired nondegenerate rigid bearing-formation $\left(\mathcal{G}, \boldsymbol{\beta}_{\mathcal{E}}^{d}\right)$, consider the relabeling and equivalent minimally-linear rigid formation $\left(\hat{\mathcal{G}}, \boldsymbol{\beta}_{\hat{\mathcal{E}}}^{d}\right)$ obtained by applying Lemma 2. Control (20), together with (25-26) and (27-29), asymptotically and almost globally steers $(\hat{\mathcal{G}}, \boldsymbol{q}(t))$ towards the particular realization of $\left(\hat{\mathcal{G}}, \boldsymbol{\beta}_{\hat{\mathcal{E}}}^{d}\right)$ such that

$$
\begin{aligned}
\boldsymbol{q}_{1}(t) & =\boldsymbol{q}_{1}\left(t_{0}\right)+\int_{t_{0}}^{t}\left(\begin{array}{c}
R\left(\psi_{1}\right) \boldsymbol{\nu} \\
w
\end{array}\right) d t \\
\delta_{12}(t) & =\delta_{12}\left(t_{0}\right)+\int_{t_{0}}^{t} s d t,
\end{aligned}
$$

provided that $\int_{t_{0}}^{t} s d t>-\delta_{12}\left(t_{0}\right)$ and $K_{p}>s$ for any $t \geq 0$. Furthermore $\delta_{1 i} \rightarrow \gamma_{12 i}^{d} \delta_{12}$, for any $i=3 \ldots N$.

Proof. The first condition (30) is a trivial consequence of (27) and (25-26) evaluated for $i=1$. In order to show (31), we differentiate the dynamics of $\delta_{12}$ using (4) together with (25),(27-28) and $i=1, j=2$, obtaining

$$
\begin{aligned}
\dot{\delta}_{12} & =\boldsymbol{\beta}_{12}^{T}\left({ }^{1} R_{2} \boldsymbol{u}_{2}^{f}\right)+s= \\
& =K_{p} \boldsymbol{\beta}_{12}^{T}{ }^{1} R_{2}{ }^{2} R_{1}\left[\boldsymbol{\beta}_{12} \times\left(\boldsymbol{\beta}_{12}^{d} \times \boldsymbol{\beta}_{12}\right)\right]+s=s .
\end{aligned}
$$

Since the control action (25-26) is designed to not change any bearing, the dynamics of $\boldsymbol{\beta}_{i j}$, obtained differentiating (3), is

$$
\dot{\boldsymbol{\beta}}_{i j}=-\left(\begin{array}{c}
0 \\
0 \\
w_{i}^{f}
\end{array}\right) \times \boldsymbol{\beta}_{i j}+\frac{I-\boldsymbol{\beta}_{i j} \boldsymbol{\beta}_{i j}^{T}}{\delta_{i j}}\left({ }^{i} R_{j} \boldsymbol{u}_{j}^{f}-\boldsymbol{u}_{i}^{f}\right) .
$$

In order to prove that $(\hat{\mathcal{G}}, \boldsymbol{q}(t))$ converges to the set of realizations of $\left(\hat{\mathcal{G}}, \boldsymbol{\beta}_{\hat{\mathcal{E}}}^{d}\right)$, it is sufficient to show that $\boldsymbol{\beta}_{i j}(t) \rightarrow \boldsymbol{\beta}_{i j}^{d}$, $\forall(i, j) \in \hat{\mathcal{E}}$. We decompose the proof among the following subcases:

a) Convergence of $\boldsymbol{\beta}_{1 i}$ to $\boldsymbol{\beta}_{1 i}^{d}$ : we first prove that $\boldsymbol{\beta}_{12} \rightarrow$ $\boldsymbol{\beta}_{12}^{d}$ by plugging the control inputs (27-28) into (32) (with $i=1, j=2$ ) and obtaining

$$
\begin{aligned}
\dot{\boldsymbol{\beta}}_{12} & =\left(K_{p} / \delta_{12}(t)\right)\left(\boldsymbol{\beta}_{12} \times\left(\boldsymbol{\beta}_{12}^{d} \times \boldsymbol{\beta}_{12}\right)\right)= \\
& =\left(K_{p} / \delta_{12}(t)\right)\left[\boldsymbol{\beta}_{12}^{d}\left(\boldsymbol{\beta}_{12}^{T} \boldsymbol{\beta}_{12}\right)-\boldsymbol{\beta}_{12}\left(\boldsymbol{\beta}_{12}^{T} \boldsymbol{\beta}_{12}^{d}\right)\right] .
\end{aligned}
$$

Consider the dynamics of the error term $e_{12}=\boldsymbol{\beta}_{12}^{T} \boldsymbol{\beta}_{12}^{d}-1$, that is,

$$
\begin{aligned}
\dot{e}_{12} & =\dot{\boldsymbol{\beta}}_{12}^{T} \boldsymbol{\beta}_{12}^{d}=-\left(K_{p} / \delta_{12}(t)\right)\left(\left(\boldsymbol{\beta}_{12}^{T} \boldsymbol{\beta}_{12}^{d}\right)^{2}-1\right)= \\
& =-\left(K_{p} / \delta_{12}(t)\right)\left(\boldsymbol{\beta}_{12}^{T} \boldsymbol{\beta}_{12}^{d}-1\right)\left(\boldsymbol{\beta}_{12}^{T} \boldsymbol{\beta}_{12}^{d}+1\right)= \\
& =-\left(K_{p} / \delta_{12}(t)\right)\left(\boldsymbol{\beta}_{12}^{T} \boldsymbol{\beta}_{12}^{d}+1\right) e_{12} .
\end{aligned}
$$

Except from the zero-measure case of an initial condition $\boldsymbol{\beta}_{12}\left(t_{0}\right)=-\boldsymbol{\beta}_{12}^{d}$, it is $\boldsymbol{\beta}_{12}^{T} \boldsymbol{\beta}_{12}^{d}+1>0$. Since also $\delta_{12}(t)>0$ by assumption, we obtain that $e_{12} \rightarrow 0$ almost everywhere and, therefore, that $\boldsymbol{\beta}_{12} \rightarrow \boldsymbol{\beta}_{12}^{d}$.

To prove that $\boldsymbol{\beta}_{1 i} \rightarrow \boldsymbol{\beta}_{1 i}^{d}$, with $i=3 \ldots N$, we show that $\boldsymbol{\beta}_{1 i} \delta_{1 i} \rightarrow \boldsymbol{\beta}_{1 i}^{d} \gamma_{12 i}^{d} \delta_{12}$, which also implies $\delta_{1 i} \rightarrow \gamma_{12 i}^{d} \delta_{12}$, i.e., $\gamma_{12 i} \rightarrow \gamma_{12 i}^{d}$. Consider now the error $e_{1 i}=\boldsymbol{\beta}_{1 i} \delta_{1 i}-$ $\boldsymbol{\beta}_{1 i}^{d} \gamma_{12 i}^{d} \delta_{12}$. Since $\boldsymbol{\beta}_{1 i} \delta_{1 i}={ }^{1} R \boldsymbol{p}_{1 i}$, after some straightforward algebra the error dynamics results in:

$$
\begin{aligned}
\dot{e}_{1 i} & ={ }^{1} R R_{i} \boldsymbol{u}_{i}^{f}-\left(\boldsymbol{\beta}_{1 i}^{d} \gamma_{12 i}^{d}-\boldsymbol{\beta}_{1 i} \gamma_{12 i}\right) s= \\
& =\left(K_{p}-s\right){ }^{1} R_{i}{ }^{i} R_{1}\left(\gamma_{12 i}^{d} \boldsymbol{\beta}_{1 i}^{d}-\gamma_{12 i} \boldsymbol{\beta}_{1 i}\right)=-\frac{K_{p}-s}{\delta_{12}(t)} e_{1 i},
\end{aligned}
$$

thus proving global exponential convergence of $e_{1 i}$ to zero since $\delta_{12}(t)>0$ and $K_{p}-s>0$ by assumption.

b) Convergence of $\boldsymbol{\beta}_{i 1}$ to $\boldsymbol{\beta}_{i 1}^{d}$ : we start showing that ${ }^{i} R_{1}$ converges to ${ }^{i} R_{1}^{d}$. Let $\psi_{1 i}=\psi_{1}-\psi_{i}$ represent the relative yaw among agents 1 and $i$, and note that, because of their definitions, ${ }^{i} R_{1}=R_{z}\left(\psi_{1 i}\right)$ and ${ }^{i} R_{1}^{d}=R_{z}\left(\psi_{1 i}^{d}\right)$. Therefore $\psi_{1 i} \rightarrow \psi_{1 i}^{d}$ implies ${ }^{i} R_{1} \rightarrow{ }^{i} R_{1}^{d}$. Define $e_{\psi_{1 i}}=\psi_{1 i}^{d}-\psi_{1 i}$ as error term and note that $\dot{e}_{\psi_{1 i}}=-w_{i}^{f}$ since $w_{1}^{h}=w_{i}^{h}=w$ from (26). By applying control (29) we obtain

$$
\dot{e}_{\psi_{1 i}}=-K_{\omega}\left[{ }^{1} R_{i}^{d i} R_{1}-{ }^{1} R_{i}{ }^{i} R_{1}^{d}\right]_{\vee, 3}=-2 K_{\omega} \sin \left(e_{\psi_{1 i}}\right) \text {, }
$$

showing that $e_{\psi_{1 i}} \rightarrow 0$ for all initial conditions apart from the zero-measure case $e_{\psi_{1 i}}\left(t_{0}\right)= \pm \pi$.

Since $\boldsymbol{\beta}_{i 1}=-{ }^{i} R_{1} \boldsymbol{\beta}_{1 i}$, and we proved that $\boldsymbol{\beta}_{1 i} \rightarrow \boldsymbol{\beta}_{1 i}^{d}$ (previous point) and ${ }^{i} R_{1} \rightarrow{ }^{i} R_{1}^{d}$, we obviously have that $\boldsymbol{\beta}_{i 1} \rightarrow \boldsymbol{\beta}_{i 1}^{d}$.

c) Convergence of $\boldsymbol{\beta}_{j k}$ to $\boldsymbol{\beta}_{j k}^{d}$, for any remaining $(j, k) \in$ $\hat{\mathcal{E}}$ : from the proof of Property 1 and Property 3 with $l=1$, $m=k, l=j$, we know that

$$
\boldsymbol{\beta}_{j k}=-{ }^{j} R_{k} \boldsymbol{\beta}_{k j}=-{ }^{j} R_{1} \frac{\boldsymbol{\beta}_{1 j} \gamma_{1 k j}-\boldsymbol{\beta}_{1 k}}{\left\|\boldsymbol{\beta}_{1 j} \gamma_{1 k j}-\boldsymbol{\beta}_{1 k}\right\|} .
$$

Note that from (8) it follows $\gamma_{1 k j}=\gamma_{12 j} / \gamma_{12 k}$. Since from the previous points, we have shown that $\left(\boldsymbol{\beta}_{1 j}, \boldsymbol{\beta}_{1 k},{ }^{j} R_{1}, \gamma_{12 j}, \gamma_{12 k}\right)$ converge to their desired values, it obviously follows that $\boldsymbol{\beta}_{j k} \rightarrow \boldsymbol{\beta}_{j k}^{d}$.

In order to better understand the action of control (27-29) it is useful to highlight, with the help of Fig. 2, some properties depending on the high-level steering inputs $\boldsymbol{\nu}, s$, and $w$ :

1) the translational dynamics of agent 1 is affected only by the high-level command $\nu$, therefore it will stay fixed in space when $\boldsymbol{\nu}=\mathbf{0}$;

2) if $s=0$ then agent 2 will rotate and travel along the geodesic path on the sphere centered on agent 1 by keeping the 'radius' $\delta_{12}$ constant. Indeed, $i$ ) the control action $\boldsymbol{u}_{2}^{f}$ is always orthogonal to $\boldsymbol{\beta}_{12}(\boldsymbol{q})$ which represents the direction of the line connecting agents 1 


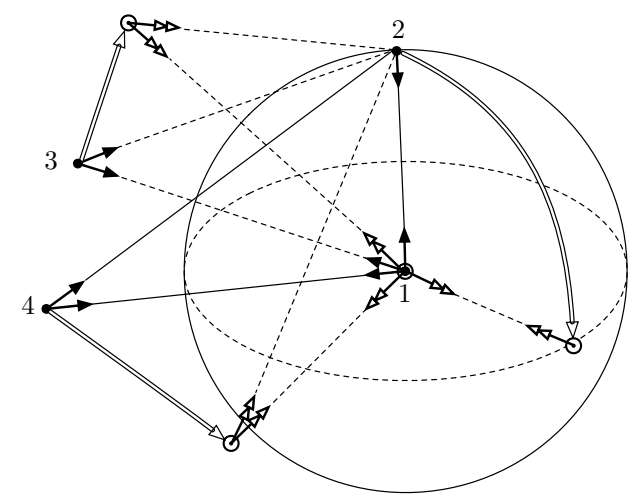

Fig. 2: Visual representation of the action of the formation controller (27-29) in the case of 4 agents. Current agent positions are marked with solid dots, while black arrows and double-white arrows denote measured and corresponding desired bearings, respectively. The target positions for every agent are marked with white circles (note that these are not explicitly computed by the controller). Long and thick white arrows indicate the paths followed by the agents under the control action: agent 1 is stationary; agent 2 moves on the sphere centered around 1 following a geodesic path; agent 3 and 4 move along a straight line. All the motions are executed without resorting to any distance measurement.

and 2 ; ii) the external input $\boldsymbol{\nu}$ moves the agents 1 and 2 cohesively, and iii) the external input $w$ generates a motion that is also orthogonal to $\boldsymbol{\beta}_{12}(\boldsymbol{q})$;

3) if $\boldsymbol{\nu}=\mathbf{0}$ and $s=w=0$ then agents $3 \ldots N$ will rotate and move along the straight lines connecting their initial position with the final position satisfying the constraints $\boldsymbol{\beta}_{i j}(\boldsymbol{q})=\boldsymbol{\beta}_{i j}^{d}, \forall(i, j) \in \hat{\mathcal{E}}, \boldsymbol{q}_{1}=\boldsymbol{q}_{1}\left(t_{0}\right)$, and $\delta_{12}=$ $\delta_{12}\left(t_{0}\right)$.

Note also that the measured quantities needed to implement controller (27-29) are $\boldsymbol{\beta}_{21}, \boldsymbol{\beta}_{1 i},{ }^{i} R_{1}$, and $\gamma_{12 i} \forall i=2 \ldots N$. Since ${ }^{i} R_{1}$ and $\gamma_{12 i}$ can always be obtained as a function of relative bearings (see Properties 1 and 2), the control law (2729) uses only relative bearings as expected. Finally we note that condition $K_{p}>s$ does not constitute a limit of the proposed controller, since it can be always guaranteed by choosing a $K_{p}$ large enough, given the range of variation of $s$ in the specific application. Consider also that in practical applications $s$ cannot grow unbounded, mainly because of the actuation limits of a real UAV.

For a generic desired bearing-formation (i.e., whose realizations have no special alignments), any triplet of agents can be chosen as beacon agents in Lemma 2 . It is then interesting to study the problem of optimizing this choice w.r.t. any suitable criterion, such as robustness against measurement noise. This point is anyway left open for future developments.

Finally, we note that control (27-29) becomes singular only when all the position of the agents happen to be aligned during a transient phase (this is structurally avoided at steadystate since $\left(\mathcal{G}, \boldsymbol{\beta}_{\mathcal{E}}^{d}\right)$ is assumed to be non-degenerate). Such situation represents a zero-measure case, and, in practice, is very unlikely to occur. For instance, we never encountered it during our simulations or experiments. Nevertheless, since it is in principle possible to fall in its neighborhood, a practical workaround is to apply a suitable constant control action for a short phase in order to quickly exit from this singular configuration.

\section{Computational and Communication complexity}

Proposition 3 (Computational Complexity). The computational complexity of control (27-29) is $O(N)$. In fact, it can be implemented by only using the $3 N-4$ relative-bearing measurements defined by the pairs included in $\hat{\mathcal{E}}$ obtained by applying Lemma 2 with $\boldsymbol{\beta}_{\mathcal{E}}^{d}$ playing the role of $\boldsymbol{\alpha}$.

Proof. Matrix ${ }^{2} R_{1}$ can be computed from $\boldsymbol{\beta}_{12}$ and $\boldsymbol{\beta}_{21}$, and ${ }^{i} R_{1}$ and $\gamma_{12 i}, i=3 \ldots N$, can be obtained as follows: i) if $i \in$ $\mathcal{I}_{2},{ }^{i} R_{1}={ }^{i} R_{2}{ }^{2} R_{1}$ and ${ }^{i} R_{2}$ can be evaluated by first applying Property 3 and then Property 1 . As for $\gamma_{12 i}$, it can be obtained by applying Property 2; ii) if $i \in \mathcal{I}_{3},{ }^{i} R_{1}={ }^{i} R_{3}{ }^{3} R_{1}$ and ${ }^{i} R_{3}$ follows by first applying Property 3 and then Property 1 . Furthermore, from its definition, $\gamma_{12 i}=\gamma_{13 i} / \gamma_{123}$, and $\gamma_{13 i}$, $\gamma_{123}$ follow from Property 2.

The applicability of Properties 1-3 is always verified at the desired bearing-formation and, thus, when the controller (2729) has reached a steady-state regime. However, during initial transients, some of the assumptions needed by the Properties could temporarily not be met, e.g., when, for $i \in \mathcal{I}_{2}, \boldsymbol{\beta}_{12}=$ $\pm \boldsymbol{\beta}_{1 i}$. These transient situations can always be disambiguated by (temporarily) exploiting additional measurements in order to recover the loss of information, see for instance the second parts of Properties 1-2.

As for the issue of communication complexity of controller (27-29), let $N_{C}$ be the number of exchanged messages per unit of time. Since not all the needed measurements are locally available, the agents need to share some information among themselves. For example, in order to implement (29), agent 2 needs to receive $\boldsymbol{\beta}_{12}$ from agent 1 . When considering inter-agent communication issues, in order to prevent network congestions it is important to render $N_{C}$ bounded w.r.t. the number of agents $N$. Typically, $N_{C}=O(N)$ is considered as a good tradeoff, see for instance the case of consensus/agreement algorithms with a bounded number of neighbors per agent [42].

Proposition 4 (Communicational Complexity). The communication complexity of controller (27-29) is $O(N)$.

Proof. Agent 1 needs no external information, agent 2 needs $\boldsymbol{\beta}_{12}$, agents $i \in \mathcal{I}_{2}$ need $\boldsymbol{\beta}_{1 i}$ and $\boldsymbol{\beta}_{2 i}$, and agents $i \in \mathcal{I}_{3}$ need $\boldsymbol{\beta}_{1 i}$ and $\boldsymbol{\beta}_{3 i}$, for a total of $N_{C}=2(N-1)+1$ exchanged messages over the network per unit of time.

\section{Time-varying desired bearings}

We finally consider the case where the desired relative bearings are not constant but (known) time-varying quantities defined as $\tilde{\boldsymbol{\beta}}_{i j}^{d}(t)=\tilde{R}_{i}(t) \boldsymbol{\beta}_{i j}^{d}$, with $\tilde{R}_{i}(t)$ given. This extension will be exploited in the experiments reported in Sec. VIII-A, and is important to both overcome limited field-of-view of the relative-bearing sensor, and to allow for a possible scanning of the environment during a 3D coverage task, e.g., exploration, mapping, or surveillance.

The rotation matrices $\tilde{R}_{i}(t)$ considered here are only of the kind $R_{z}(\cdot)$ since these are the ones affected by the agent inputs $w_{i}$. Denote with $\tilde{\boldsymbol{\omega}}_{i}(t)=\left(\begin{array}{lll}0 & 0 & \tilde{\omega}_{i, 3}\end{array}\right)^{T} \in \mathbb{R}^{3}$ the angular velocity associated to $\tilde{R}_{i}(t)$, i.e., such that $\tilde{\boldsymbol{\omega}}_{i}(t)=\left[\tilde{R}_{i}^{T}(t) \dot{\tilde{R}}_{i}(t)\right]_{\vee}$, 
where the $[\cdot]_{\vee}$ represents the vector associated to a skewsymmetric matrix. Presence of a time-varying bearing can be easily handled by using $\tilde{\boldsymbol{\beta}}_{i j}$ instead of $\boldsymbol{\beta}_{i j}^{d}$ and adding a suitable feedforward term to the yaw-rate part of the controller (27-29), i.e., using:

$$
\begin{aligned}
w_{1}^{f} & =-\tilde{\omega}_{1,3} \\
w_{2}^{f} & =-\tilde{\omega}_{2,3}+K_{\omega}\left[{ }^{1} \tilde{R}_{2}{ }^{2} R_{1}-{ }^{1} R_{2}{ }^{2} \tilde{R}_{1}\right]_{\vee, 3} \\
w_{i}^{f} & =-\tilde{\omega}_{i, 3}+K_{\omega}\left[{ }^{1} \tilde{R}_{i}{ }^{i} R_{1}-{ }^{1} R_{i}{ }^{i} \tilde{R}_{1}\right]_{\vee, 3} .
\end{aligned}
$$

Note that presence of a time-varying component in the desired bearings does not affect the overall formation shape, but only causes the agents to suitably rotate in space. In this way, the agents can track any exogenous rotation signal while still keeping the same shape implicitly defined by the static component of the desired bearings.

\section{Case of a Human Co-operator: Haptic Steering}

We consider in this section the particular but relevant case in which a human co-operator is in charge of collectively steering the formation by selecting values for the quantities $\boldsymbol{\nu}, s$ and $w$ in the control action (25-26). As explained in the Introduction, in this case we also consider presence of suitable haptic cues for the human co-operator in a typical bilateral force-feedback architecture, since this design has proven to significantly increase the performances in humanrobot cooperation.

To this aim we propose a bilateral controller connecting the UAV group with two haptic interfaces: a 3-dof interface for controlling the group linear velocity $\boldsymbol{\nu}$, and a 2-dof interface for commanding the group expansion/rotation rates $(s, w)$. These haptic devices are modeled as generic mechanical systems

$$
\begin{aligned}
M_{t}\left(\boldsymbol{x}_{t}\right) \ddot{\boldsymbol{x}}_{t}+C_{t}\left(\boldsymbol{x}_{t}, \dot{\boldsymbol{x}}_{t}\right) \dot{\boldsymbol{x}}_{t}=\boldsymbol{\tau}_{t}+\boldsymbol{f}_{t} \\
M_{r}\left(\boldsymbol{x}_{r}\right) \ddot{\boldsymbol{x}}_{r}+C_{r}\left(\boldsymbol{x}_{r}, \dot{\boldsymbol{x}}_{r}\right) \dot{\boldsymbol{x}}_{r}=\boldsymbol{\tau}_{r}+\boldsymbol{f}_{r}
\end{aligned}
$$

where $\boldsymbol{x}_{t} \in \mathbb{R}^{3}$ and $\boldsymbol{x}_{r}=\left(\begin{array}{ll}x_{s} & x_{w}\end{array}\right)^{T} \in \mathbb{R}^{2}$ are the device position vectors, $M_{t}\left(\boldsymbol{x}_{t}\right) \in \mathbb{R}^{3 \times 3}$ and $M_{r}\left(\boldsymbol{x}_{r}\right) \in$ $\mathbb{R}^{2 \times 2}$ their positive-definite and symmetric inertia matrices, $C_{t}\left(\boldsymbol{x}_{t}, \dot{\boldsymbol{x}}_{t}\right) \in \mathbb{R}^{3 \times 3}$ and $C_{r}\left(\boldsymbol{x}_{r}, \dot{\boldsymbol{x}}_{r}\right) \in \mathbb{R}^{2 \times 2}$ represent Coriolis and centrifugal terms, and the pairs $\left(\boldsymbol{f}_{t}, \boldsymbol{\tau}_{t}\right) \in \mathbb{R}^{3} \times \mathbb{R}^{3}$, $\left(\boldsymbol{f}_{r}, \boldsymbol{\tau}_{r}\right) \in \mathbb{R}^{2} \times \mathbb{R}^{2}$ are the human/control forces acting on each device, respectively. As usually done, we also assume that gravity effects are locally compensated.

The control actions are implemented by setting in (25-26)

$$
\boldsymbol{\nu}=\lambda_{t} \boldsymbol{x}_{t}, \quad\left(\begin{array}{c}
s \\
w
\end{array}\right)=\left(\begin{array}{cc}
\lambda_{s} & 0 \\
0 & \lambda_{w}
\end{array}\right) \boldsymbol{x}_{r},
$$

where $\lambda_{t}>0, \lambda_{s}>0$, and $\lambda_{w}>0$ are suitable scaling factors from the device positions $\left(\boldsymbol{x}_{t}, \boldsymbol{x}_{r}\right)$ to the generalized velocity commands. The proposed architecture implements a position-velocity coupling between the haptic device and the UAV group. This is the most natural choice in order to handle the kinematic dissimilarity, i.e., the fact that the haptic device has a bounded workspace but the UAVs are characterized by an unbounded workspace, e.g., see also [29].
As for the reverse channel of the human-UAV connection, we chose to provide haptic cues informative of how well the real UAVs, as a group, are executing the desired human commands (38). Recalling Sec. II-A, we let $\dot{\boldsymbol{p}}_{\mathcal{A}_{i}} \in \mathbb{R}^{3}$ be the body-frame velocity vector of the $i$-th $\mathrm{UAV}$, and $\dot{\psi}_{\mathcal{A}_{i}} \in \mathbb{R}$ its yaw rate. We stress, again, that these represent real (measured) UAV quantities and not the reference (virtual) velocities of the agent model (2) tracked by the UAVs.

After the initial transient needed for reaching the desired bearing formation, i.e., when controller (27-29) has reached its steady-state, the components $\left(\boldsymbol{u}_{i}^{f}, w_{i}^{f}\right)$ in (20) become negligible and the only motion input for the UAV group is due to the high-level commands $\left(\boldsymbol{u}_{i}^{h}, w_{i}^{h}\right)$ (25-26). As first haptic cue, we can then consider the mismatch between the commanded translational velocity $\boldsymbol{\nu}$ and its actual execution by the UAVs. From (25) and (38) we have that, for each $i$-th UAV,

$$
\begin{aligned}
\boldsymbol{x}_{t} & =\frac{1}{\lambda_{t}} \boldsymbol{\nu}=\frac{1}{\lambda_{t}}{ }^{1} R_{i}\left(\boldsymbol{u}_{i}^{h}+s \gamma_{12 i} \boldsymbol{\beta}_{i 1}-w \delta_{12} \gamma_{12 i} S \boldsymbol{\beta}_{i 1}\right) \\
& =\frac{1}{\lambda_{t}} R_{i}\left(\boldsymbol{u}_{i}^{h}+\gamma_{12 i}\left(\lambda_{s} x_{s} \boldsymbol{\beta}_{i 1}-\lambda_{w} x_{w} \delta_{12} S \boldsymbol{\beta}_{i 1}\right)\right) \\
& \simeq \frac{1}{\lambda_{t}} R_{i}\left(\dot{\boldsymbol{p}}_{\mathcal{A}_{i}}+\gamma_{12 i}\left(\lambda_{s} x_{s} \boldsymbol{\beta}_{i 1}-\lambda_{w} x_{w} \delta_{12} S \boldsymbol{\beta}_{i 1}\right)\right)=: \boldsymbol{z}_{t i}
\end{aligned}
$$

The approximation in (39) has two sources: $(i)$ temporary presence of nonzero formation control inputs $\left(\boldsymbol{u}_{i}^{f}, w_{i}^{f}\right)$ in (20) because of transient disturbances in the maintenance of the bearing-formation, and $(i i)$ a generic non-perfect tracking of the commanded velocities $\boldsymbol{u}_{i}$ by means of the UAVs, that is, $\dot{\boldsymbol{p}}_{\mathcal{A}_{i}} \neq \boldsymbol{u}_{i}^{h}$ (e.g., presence of wind, actuator saturations, UAV inertia, etc.). Whatever the reason, the mismatch $\boldsymbol{e}_{t i}=\boldsymbol{x}_{t}-\boldsymbol{z}_{t i}$ represents a good measurement of how well the $i$-th UAV is executing the human translational motion command $\boldsymbol{\nu}$. By averaging $\boldsymbol{e}_{t i}$ over all UAVs, we get the average translational mismatch as

$$
\boldsymbol{e}_{t}=\boldsymbol{x}_{t}-\frac{1}{N} \sum_{i=1}^{N} \boldsymbol{z}_{t i}=\boldsymbol{x}_{t}-\boldsymbol{z}_{t} .
$$

Analogously, we can also consider the mismatch between commanded expansion/rotation rates $(s, w)$ and their actual execution by the UAVs. From (25) and (38) it can be seen that, for each $i$-th $\mathrm{UAV}$,

$$
\begin{aligned}
x_{s} & =\frac{s}{\lambda_{s}}=\frac{1}{\lambda_{s} \gamma_{12 i}}\left({ }^{i} R_{1} \boldsymbol{\nu}-\boldsymbol{u}_{i}^{h}+w \delta_{12} S \boldsymbol{\beta}_{i 1}\right) \cdot \boldsymbol{\beta}_{i 1}= \\
& =\frac{1}{\lambda_{s} \gamma_{12 i}}\left({ }^{i} R_{1} \boldsymbol{\nu}-\boldsymbol{u}_{i}^{h}\right) \cdot \boldsymbol{\beta}_{i 1} \simeq \frac{1}{\lambda_{s} \gamma_{12 i}}\left({ }^{i} R_{1} \boldsymbol{\nu}-\dot{\boldsymbol{p}}_{\mathcal{A}_{i}}\right) \cdot \boldsymbol{\beta}_{i 1} \\
& =: z_{s i}
\end{aligned}
$$

where we exploited the fact that $S \boldsymbol{\beta}_{i 1} \perp \boldsymbol{\beta}_{i 1}$. By averaging over all UAVs, we get the overall expansion error

$$
e_{s}=x_{s}-\frac{1}{N} \sum_{i=1}^{N} z_{s i}=x_{s}-z_{s} .
$$

As for the rotation rate, eq. (26) simply yields

$$
x_{w}=\frac{w}{\lambda_{w}} \simeq \frac{\dot{\psi}_{\mathcal{A}_{i}}}{\lambda_{w}}=z_{w i}
$$


with the corresponding average error

$$
e_{w}=x_{w}-\frac{1}{N} \sum_{i=1}^{N} z_{w i}=x_{w}-z_{w} .
$$

We can then express the mismatch between the commanded and actual expansion/rotation rates as the vector

$$
\boldsymbol{e}_{r}=\left(\begin{array}{c}
e_{s} \\
e_{w}
\end{array}\right)=\boldsymbol{x}_{r}-\left(\begin{array}{c}
z_{s} \\
z_{w}
\end{array}\right)=\boldsymbol{x}_{r}-\boldsymbol{z}_{r} .
$$

Note that an evaluation of $\boldsymbol{z}_{t}$ and $\boldsymbol{z}_{r}$ requires each UAV to send to the haptic device its body-frame velocity and yaw rate $\left(\dot{\boldsymbol{p}}_{\mathcal{A}_{i}}, \dot{\psi}_{\mathcal{A}_{i}}\right)$, its relative bearing $\boldsymbol{\beta}_{i 1}$ w.r.t. agent 1 , and the scaling factor $\gamma_{12 i}$.

The control torques in (36-37), aimed at providing a useful force-feedback to the human operator, are then computed as

$$
\begin{gathered}
\boldsymbol{\tau}_{t}=-B_{t} \dot{\boldsymbol{x}}_{t}-K_{t} \boldsymbol{x}_{t}-K_{t}^{e} \boldsymbol{e}_{t} \\
\boldsymbol{\tau}_{r}=-B_{r} \dot{\boldsymbol{x}}_{r}-K_{r} \boldsymbol{x}_{r}-K_{r}^{e} \boldsymbol{e}_{r} .
\end{gathered}
$$

Here, $B_{t} \in \mathbb{R}^{3 \times 3}, B_{r} \in \mathbb{R}^{2 \times 2}$ are positive definite damping matrixes whose role is to stabilize the haptic devices, $K_{t} \in \mathbb{R}^{3 \times 3}, K_{r} \in \mathbb{R}^{2 \times 2}$ are semi-definite diagonal matrixes (possibly null) for giving the user a perception of the distance to the zero-commanded velocity, and $K_{t}^{e} \in \mathbb{R}^{3 \times 3}, K_{r}^{e} \in \mathbb{R}^{2 \times 2}$ are positive definite diagonal matrixes meant as scaling factors for $\boldsymbol{e}_{t}$ and $\boldsymbol{e}_{r}$.

Control (46-47) is, however, not robust against the destabilizing effects of the typical non-idealities in hapticdevice/UAVs communication channels, that is, possible presence of discrete sampling, delays and packet losses. In order to guarantee teleoperation stability despite these effects, let $\left(\boldsymbol{z}_{t}[k], \boldsymbol{z}_{r}[k]\right)$ be the discrete (received) versions of $\left(\boldsymbol{z}_{t}(t), \boldsymbol{z}_{r}(t)\right)$. Then, the actual implementation of the force controller takes the form

$$
\begin{aligned}
\boldsymbol{\tau}_{t} & =-B_{t} \dot{\boldsymbol{x}}_{t}-K_{t} \boldsymbol{x}_{t}-K_{t}^{e}\left(\boldsymbol{x}_{t}-\overline{\boldsymbol{z}}_{t}[k]\right) \\
\boldsymbol{\tau}_{r} & =-B_{r} \dot{\boldsymbol{x}}_{r}-K_{r} \boldsymbol{x}_{r}-K_{r}^{e}\left(\boldsymbol{x}_{r}-\overline{\boldsymbol{z}}_{r}[k]\right) .
\end{aligned}
$$

where the quantities $\left(\overline{\boldsymbol{z}}_{t}[k], \overline{\boldsymbol{z}}_{r}[k]\right)$ are the passive set-position modulation (PSPM) versions of the received $\left(\boldsymbol{z}_{t}[k], \boldsymbol{z}_{r}[k]\right)$. The PSPM framework [43] is a general tool for guaranteeing haptic-device passivity and, therefore, stability of the closed-loop system when dealing with possible delays/discretization/packet losses of the signals exchanged over the haptic-device/UAVs communication channel. The action of the PSPM is to modulate a received signal $\boldsymbol{z}[k]$ into a (possibly) attenuated version $\overline{\boldsymbol{z}}[k]$ so that implementation of (4849) will meet the haptic-device (energetic) passivity constraint over its external power port. Being the slave considered in this work a kinematic (first-order) system (agent model (2)), this is indeed sufficient to ensure stability of the overall closedloop teleoperation scheme by further assuming, as usually done (see, e.g., [44]), passivity of the human side. We omit further details here and refer the interested reader to [28], [43] and references therein for a complete treatment and formal proofs of these statements. Note that, besides the passifying action against non-idealities of the communication channel, the PSPM framework also allows to enforce closed-loop stability despite the nonstandard position-velocity coupling between haptic-device and the UAVs adopted in our work, see again [28] for a more thorough treatment.

The resulting haptic cues will not only give a general feeling of the execution accuracy of the human commands, but are specifically designed to represent the execution mismatches along the 5 motion directions in a decoupled way.

\section{Overall System Architecture}

For the reader's convenience, Fig. 3 summarizes the overall conceptual scheme of our teleoperation system from the perspective of the generic $i$-th UAV in the group. At the beginning of the task, the high-level planner (lower/left-side, e.g., an autonomous exploration algorithm or a human co-operator) selects and communicates the desired bearing-formation to the $i$-th UAV. Then, during the task execution, it sends to the $i$-th UAV the commands $(\boldsymbol{\nu}, s, w)$ in (38). In the case of a human co-operator, this is done by acting on the haptic devices, and he/she is provided with the haptic cues (48-49). The generic UAV computes the desired velocities $(\boldsymbol{u}, w)$ as the sum of the formation control terms in (27-29) and of the high-level steering terms (25-26) (center of the scheme). The velocity tracker then regulates the real (measured) UAV velocities $\left(\dot{\boldsymbol{p}}_{\mathcal{A}}, \dot{\psi}_{\mathcal{A}}\right)$ to the desired ones. These measured velocities are also sent back to the haptic interface (if present) in order to realize the haptic cues. An additional module measures the UAV relative bearings $\boldsymbol{\beta}_{\mathcal{A}_{i} \mathcal{A}_{j}}$ and roll/pitch angles $\left(\phi_{\mathcal{A}_{i}}, \theta_{\mathcal{A}_{i}}\right)$ to obtain the corresponding inter-agent relative bearings (5) which are then used by the UAV controller and communicated to the haptic device (if present). Finally, the needed relative bearings are also communicated to the other UAVs in the group (upper/left-side).

\section{EXPERIMENTAL TESTBED}

In the simulations and experiments we opted for having a human co-operator as high-level planner instead of an autonomous algorithm. This allows to illustrate and validate all the parts of our framework, i.e., including the haptic component. The two haptic devices used both for the simulations and real experiments are shown in Fig. 4(a): the device on the right is the 3-dof device ${ }^{3}$ modeled by (36) and responsible for the command $\nu$ in (38) and the force feedback term $\tau_{t}$ in (48). The device on the left is also a 3-dof device but constrained via software to only move on the $2 \mathrm{D}$ horizontal plane in order to behave as (37). This device is responsible for the commands $(s, w)$ in (38) and the force feedback term $\boldsymbol{\tau}_{r}$ in (49). Both devices are connected to a GNU-Linux machine where a local control loop implements the forces $\tau_{t}$ and $\tau_{r}$ at a frequency of $2.5 \mathrm{kHz}$. The control program also sends over a wireless channel the inputs $(\boldsymbol{\nu}, s, w)$ to the UAV controllers at $120 \mathrm{~Hz}$, and receives the UAV measurements needed to implement $\tau_{t}$ and $\boldsymbol{\tau}_{r}$.

The UAVs used in these simulations/experiments are quadrotors, see Figs. 4-5. Indeed, the use of quadrotors makes it possible to empirically validate our preliminary assumption of Sec. II, i.e., to exactly track the virtual agent dynamics (2)

\footnotetext{
${ }^{3}$ http://www.forcedimension.com
} 


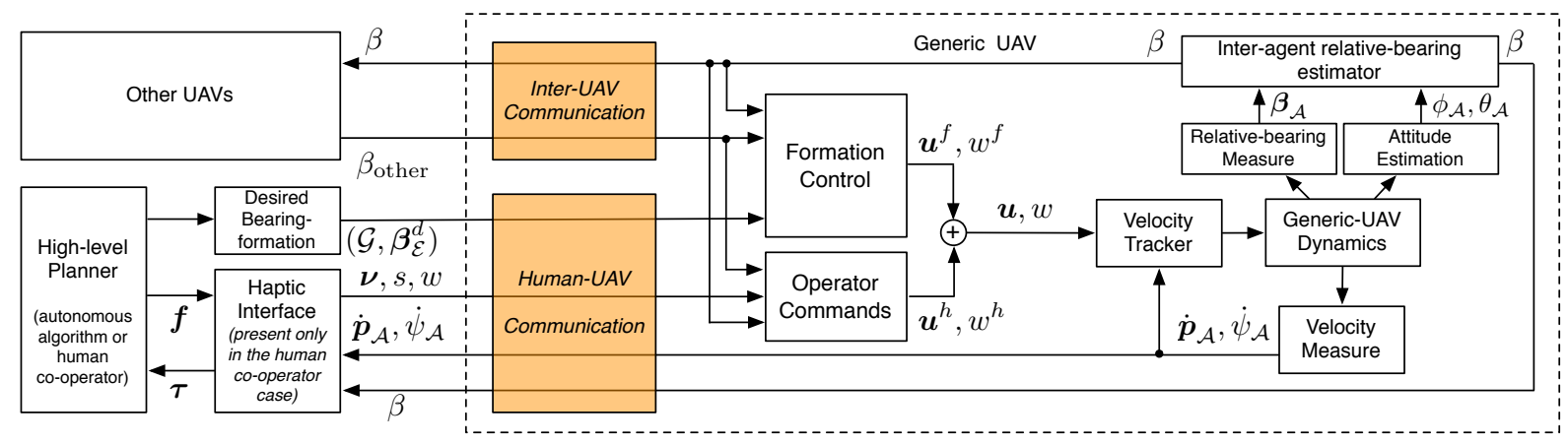

Fig. 3: Overall system architecture as seen from the point-of-view of a generic UAV.

thanks to the flatness of the quadrotor outputs $\left(\boldsymbol{p}_{\mathcal{A}_{i}}, \psi_{\mathcal{A}_{i}}\right)$. The controller of each quadrotor (both simulated/real) runs two processes: the agent-process and the velocity-tracker. The velocity-tracker enables the quadrotor to track smooth bodyframe reference velocities $\left(\boldsymbol{u}_{i}, w_{i}\right)$ implementing a standard cascaded controller similar to the one used in [45] but without position error terms. These reference velocities are in turn generated by the agent-process, which communicates via a wireless link with the agent processes of the other UAVs and with the controller of the haptic devices in order to eventually compute the (control) velocity terms $\left(\boldsymbol{u}_{i}, w_{i}\right)$ in (20).

Our simulation environment is made of a custom software [46] based on third party 3D graphics and physics engines ${ }^{4}$ (see Fig. 4(b) for two screenshots). This environment simulates the quadrotor rigid-body dynamics and generates the measurements needed by the controllers.

As for the experiments, we used 3 real quadrotors ${ }^{5}$ whose setup is shown in Fig. 5(a). The velocity-tracker and agentprocess implementation is split between the onboard microcontroller (Fig. 5(c1)) and a small GNU-Linux PC-board ${ }^{6}$ mounted underneath the quadrotor (Fig. 5(c3)). Measurements of the quadrotor current roll and pitch angles $\left(\phi_{\mathcal{A}_{i}}, \theta_{\mathcal{A}_{i}}\right)$ are obtained fusing the onboard IMU readings by means of a standard complementary filter. The absolute yaw $\psi_{\mathcal{A}_{i}}$, on the other hand, is not measured nor estimated since it is not needed by our approach. We further used an external optical tracking system ${ }^{7}$ in order to only retrieve the quadrotor body-frame velocity since, again, no additional global position measurements were needed. This velocity information could also be obtained exploiting vision/optical flow [47] or range finders.

The relative bearings needed by our controller were obtained from an onboard monocular camera (Fig. 5(b)) with an horizontal/vertical FOV of about $88 / 60 \mathrm{deg}$. In particular, by equipping every quadrotor with a colored sphere on its top (Fig. 5(a1)), measurements of relative bearings are estimated by segmenting these spheres from the onboard camera images. This algorithm could run with a rate of about $7 \mathrm{~Hz}$ and with an average latency of $500 \mathrm{~ms}$. This relatively poor performance was mainly due to the absence of a dedicated driver for the

\footnotetext{
${ }^{4}$ http://www.ogre3d.org, http://www.nvidia.com/object/physx_new.html ${ }^{5}$ http://www.mikrokopter.de

${ }^{6} \mathrm{http}: / / \mathrm{www} . \operatorname{seco.it/en/,~http://www.qseven-standard.org/~}$

${ }^{7}$ http://www.vicon.com/
}
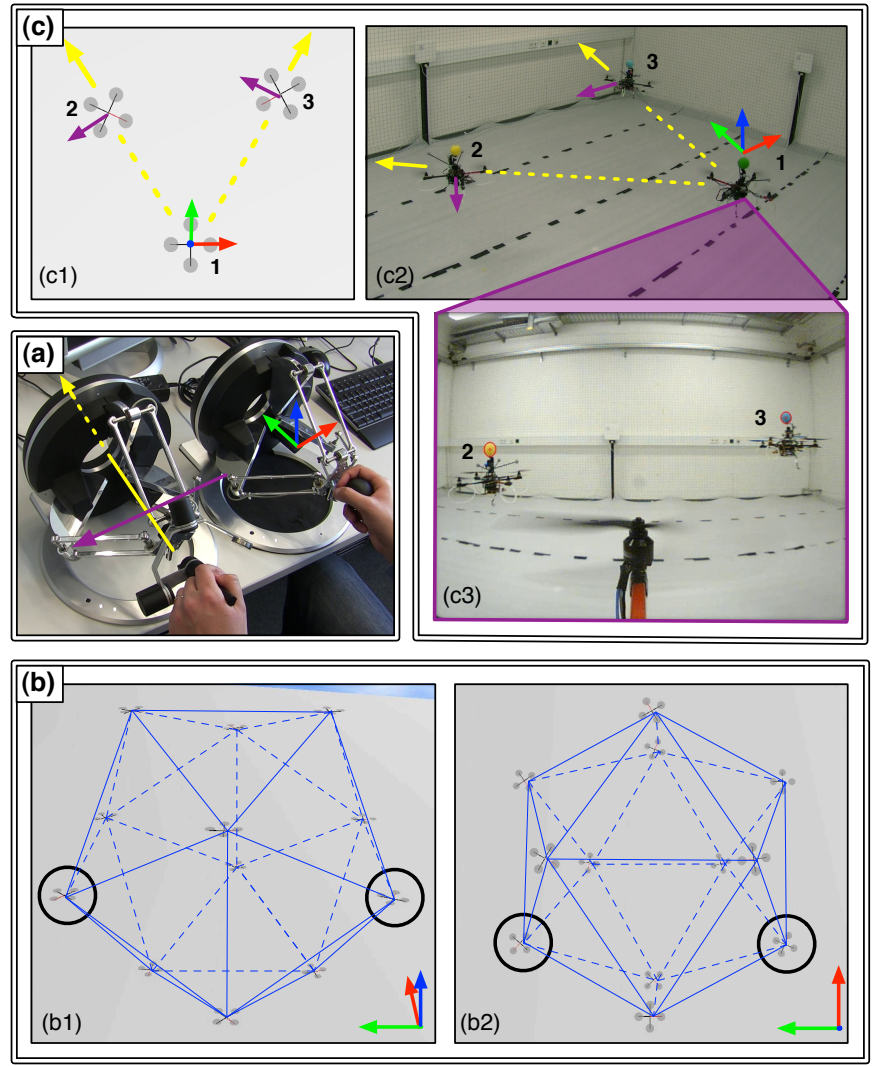

Fig. 4: Experimental setup: (a) 3-dof haptic-feedback devices used to perform the bilateral high-level steering. (b) Simulation environment used to physically simulate the quadrotors: side (b1) and top (b2) views of 12 quadrotors in a dodecahedron formation with agents 1 and 2 being highlighted. (c) Real UAV setup with 3 quadrotors: (c1) top-view of the formation in the 3D visualizer; (c2) triangular formation used during the experiments; (c3) onboard camera view of agent 1 with agent 2 and 3 detected by the image processing algorithm.

camera. Nevertheless, as it will be clear form the experiments, our approach was robust enough to deal with these and additional non-idealities representative of real-world conditions. A visualization of the ball-tracker output is shown in Fig. 4(c3), where the detection of the balls is highlighted with red circles.

\section{Simulations AND EXPERIMENTS}

We first present the results of a Human/Hardware in-theloop (HHIL) simulation involving 12 UAVs starting far from 

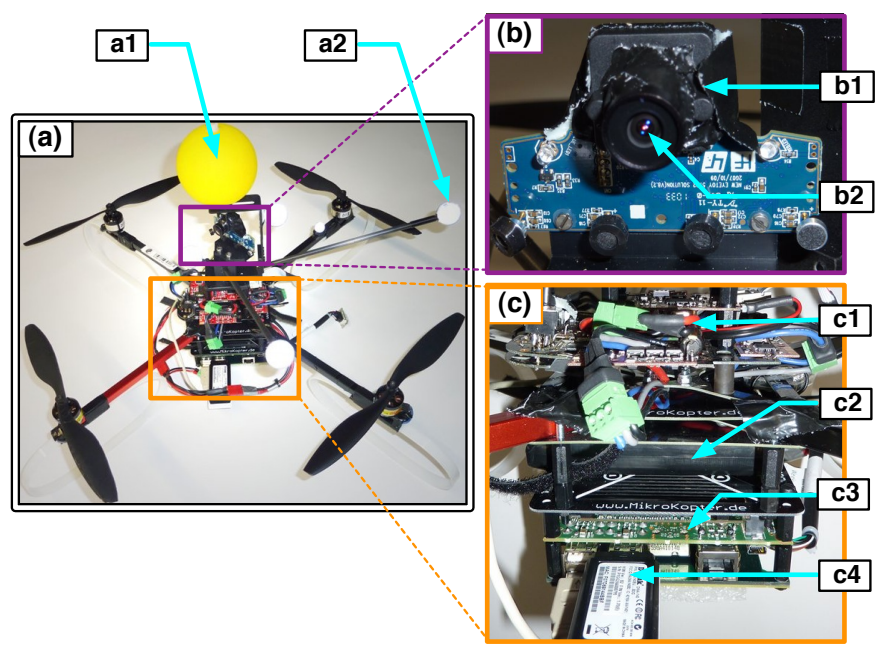

Fig. 5: Quadrotor setup. (a) Quadrotor in its flight configuration: a1) colored sphere used for the visual tracking, a2) reflective marker used for the ground-truth tracking system (b) Camera setup: b1) Consumermarket camera, b2) $140^{\circ}$ lens. (c) Computational setup: c1) Microcontroller and IMU, c2) Battery, c3) GNU-Linux PC Board, c4) Wireless adapter.

the desired bearing-formation. In this simulation we assumed an unlimited FOV capability for the UAVs so that relative bearings could always be retrieved.

The simulation is articulated in two phases: at the beginning, only the formation control is active while the human operator holds the two haptic devices fixed at their neutral position. After the time $t=17 \mathrm{~s}$ (vertical dashed black line in Figs. 6(af)), the desired formation is eventually reached, and the human operator starts commanding the overall group. This second phase is itself split into two sub-phases: at first (from $t=17 \mathrm{~s}$ to $t=110 \mathrm{~s}$ ), the human operator intentionally commands the 5 available motion directions once at a time by following this particular order $\boldsymbol{\nu}_{y} \rightarrow \boldsymbol{\nu}_{x} \rightarrow \boldsymbol{\nu}_{z} \rightarrow r \rightarrow w$ (see Fig. 6(b)). This is done so as to isolate the effects of each command. Then, during the last sub-phase (from $t=110 \mathrm{~s}$ to $t=150 \mathrm{~s}$ ), the human operator gives a generic command which shuffles the 5 motion directions all together.

Figures $6(a)-(c)$ show the average tracking errors between the actual UAV velocities $\left(\dot{\boldsymbol{p}}_{\mathcal{A}_{i}}, \dot{\psi}_{\mathcal{A}_{i}}\right)$ and the reference velocities $\left(\boldsymbol{u}_{i}, w_{i}\right)$ associated to the agent model (2). As expected, due to the flatness of the quadrotor, the velocity tracker is able to keep the tracking error sufficiently small, thus confirming the assumptions of Sec. II. Figure 6(e), shows the evolution of the average quadratic error of the bearing-formation $\boldsymbol{\beta}_{i j}^{d}-\boldsymbol{\beta}_{i j}$, $\forall \boldsymbol{\beta}_{i j}^{d} \in \boldsymbol{\beta}_{\mathcal{E}}^{d}$ : this goes exponentially to zero until $t=17 \mathrm{~s}$, and then does not increase when the human starts commanding the group during the second phase. The only exception is a bounded error occurring whenever the human is commanding a rotation $w$ : this is due to the mismatch between the actual distance $\delta_{12}$ and the constant guess $\hat{\delta}_{12}$ used to implement (25), see the remarks in Sec. IV-A. This is in perfect agreement with our theoretical analysis and expectations.

Figure 6(d) shows the behavior of the error vectors $\left(\boldsymbol{e}_{t}, \boldsymbol{e}_{r}\right)$ in (40)-(45). The peaks occur when the commanded acceleration is at its maximum as a consequence of the non-negligible

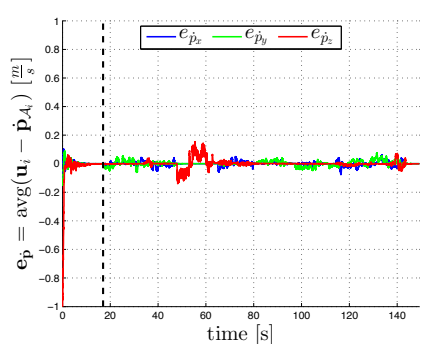

(a)

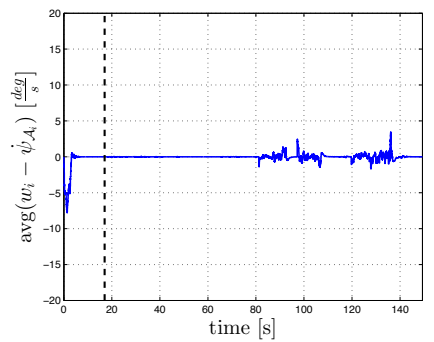

(c)

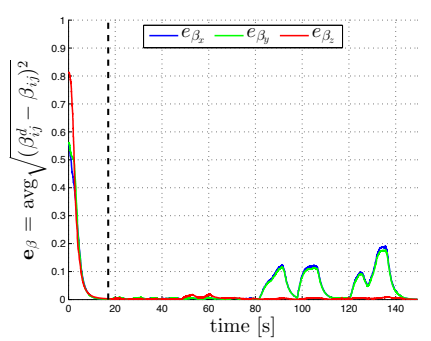

(e)

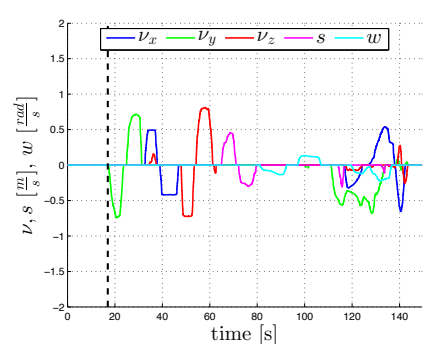

(b)

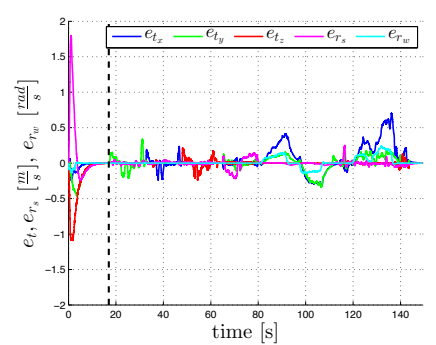

(d)

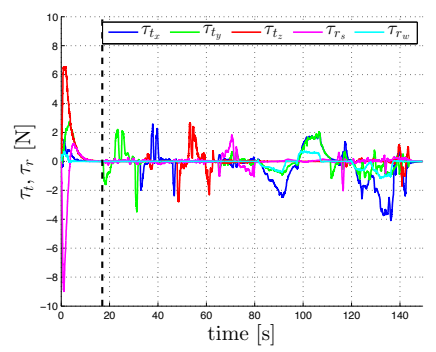

(f)
Fig. 6: Results with a group of 12 simulated quadrotors. (a),(c): Average velocity and yaw rate tracking errors; (b): Operator commands: translation velocity, expansion rate and rotation speed; (d): Mismatch between commands and executions; (f): Force feedback; (e): Mean square error w.r.t. desired bearing-formation.

inertia of the physically simulated quadrotors. Note also how the components of $\left(\boldsymbol{e}_{t}, \boldsymbol{e}_{r}\right)$ show a good decoupling during the first phase of the human operation, roughly from $t=17 \mathrm{~s}$ to $t=110 \mathrm{~s}$ : activation of one particular command among $(\boldsymbol{\nu}, r, w)$ only affects its associated component of the error vectors $\left(\boldsymbol{e}_{t}, \boldsymbol{e}_{r}\right)$, with, again, the only exception of the command $w$ because of the wrong estimate $\hat{\delta}_{12}$. Finally, Fig. 6(e) shows the control torques $\left(\boldsymbol{\tau}_{t}, \boldsymbol{\tau}_{r}\right)$ computed from (48-49) during the motion. These are basically proportional to the error vectors $\left(\boldsymbol{e}_{t}, \boldsymbol{e}_{r}\right)$ as expected, since matrices $K_{t}^{e}$ and $K_{r}^{e}$ have been chosen to be dominant w.r.t to $B_{t}, K_{t}$, and $B_{r}, K_{r}$, respectively, in (46-47).

We report now the results of an experiment run with the 3 real quadrotors and the setup shown in Figs. 4-5. In this case, the quadrotors start being already close to the desired bearing-formation and the human operator can control their motion from the beginning of the experiment. Screenshots of the experiments at three different times $t_{1}=36 \mathrm{~s}, t_{2}=62 \mathrm{~s}$ and $t_{3}=79 \mathrm{~s}$ are shown in Fig. 7. Since the cameras onboard the quadrotors have a limited FOV, we carefully selected the desired bearing-formation such that every UAV is in the visibility range of the others.

In Figs. 8(a)-(c) it can be again observed that the average tracking errors of the quadrotor actual velocities $\left(\dot{\boldsymbol{p}}_{\mathcal{A}_{i}}, \dot{\psi}_{\mathcal{A}_{i}}\right)$ 


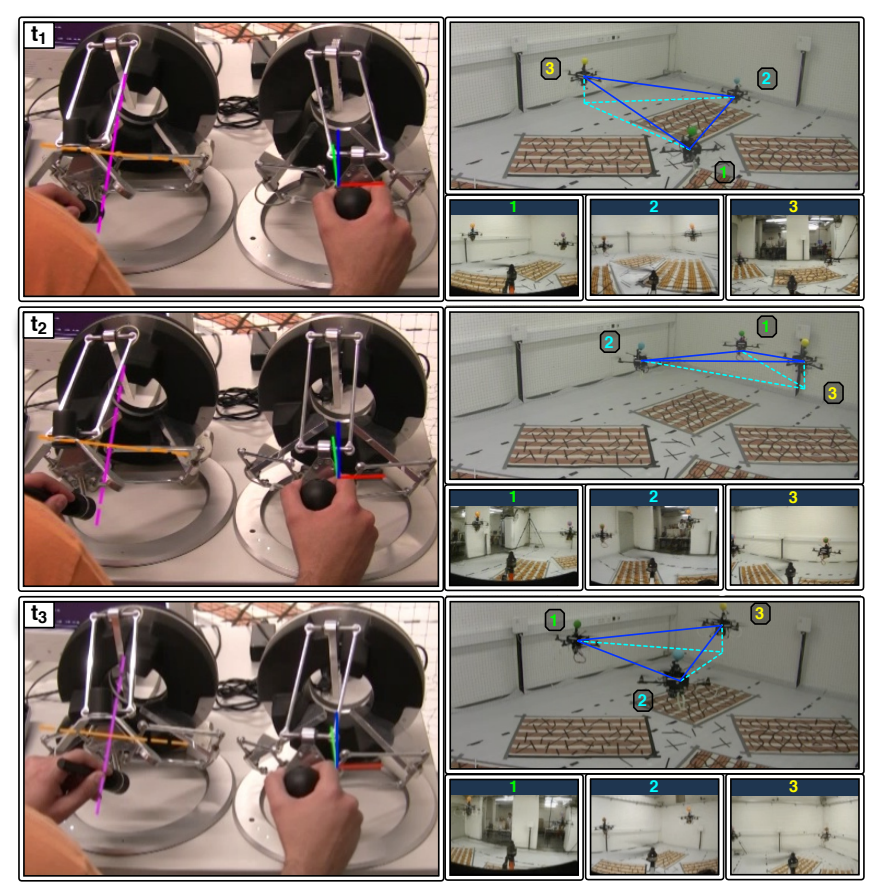

Fig. 7: Three snapshots of the experiment with 3 quadrotors . Pictures on the left show the haptic interfaces used to command the group motion and receive suitable haptic cues. Pictures on the right report the corresponding external views of the formation and, superimposed, the local views from the onboard cameras of each UAV.

w.r.t. the reference velocities $\left(\boldsymbol{u}_{i}, w_{i}\right)$ stays approximately zero despite the noisier measurements and all the unmodeled dynamics not taken into account in the analysis and simulation results - see Figs. 6(a)-(c) for a comparison. Similarly, Fig. 8(e) shows that the average quadratic bearing error also remains approximately zero during the motion. A bounded error only appears when the human operator commands a rotation of the formation for the reasons explained before, thus confirming again the theoretical analysis and the simulation results. The behavior of the user commands and error vectors $\left(e_{t}, e_{r}\right)$ is reported in Figs. 8(b)-(d), respectively. Once again, note the bounded translational error triggered by the rotation command to the whole formation. Finally, Fig. 8(f) shows the control torques $\left(\boldsymbol{\tau}_{t}, \boldsymbol{\tau}_{r}\right)$ presented as force cues to the human operator.

Onboard cameras provide the position of the tracked object in their image plane, i.e., an information equivalent to a pair of horizontal/vertical angles (azimuth and elevation). Relative bearings $\boldsymbol{\beta}_{i j}$ can then be computed in terms of relative azimuth $\zeta_{i j}$ and elevation $\eta_{i j}$ as

$$
\boldsymbol{\beta}_{i j}=\left(\begin{array}{lll}
\cos \eta_{i j} \cos \zeta_{i j} & \cos \eta_{i j} \sin \zeta_{i j} \sin \eta_{i j}
\end{array}\right)^{T} .
$$

Figure 9(a) shows the mean square error between the azimuth/elevation measurements obtained from the onboard cameras and those obtained from the ground-truth. One can verify that the mismatch always stays below $3 \mathrm{deg}$. We also note a constant temporal lag of 0.5 , w.r.t. the ground-truth, as can be seen from Fig. 9(b). Nevertheless, our control framework proved to be robust enough to this unmodeled delay.

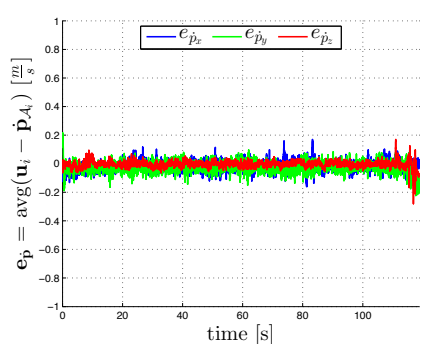

(a)

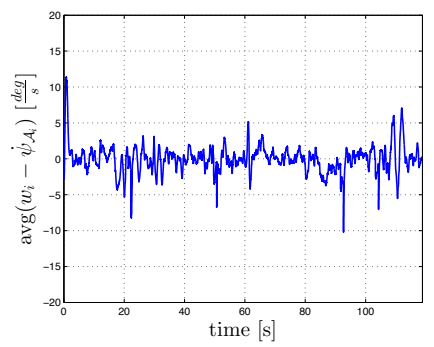

(c)

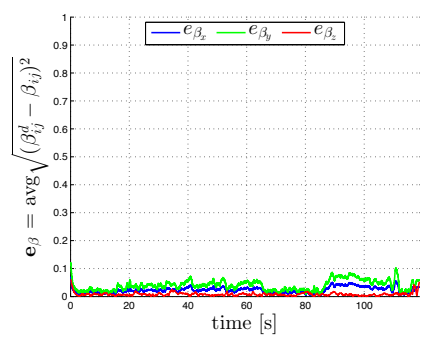

(e)

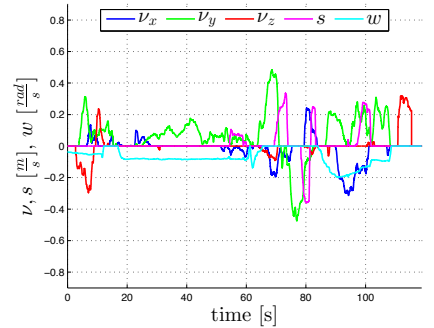

(b)

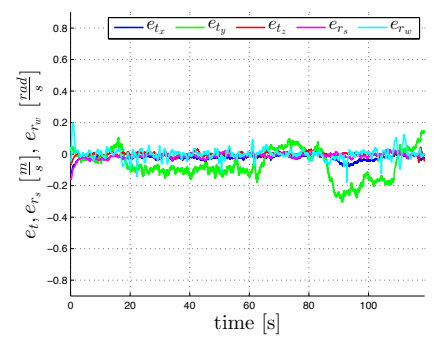

(d)

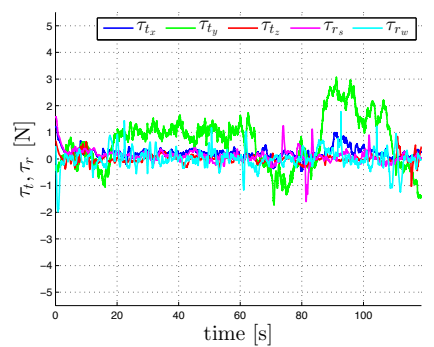

(f)
Fig. 8: Results with a group of 3 real quadrotors. (a),(c): Average velocity and yaw rate tracking errors; (b): Operator commands: translation velocity, expansion rate and rotation speed; (d): Mismatch between commands and executions; (f): Force feedback; (e): Mean square error w.r.t. desired bearing-formation.

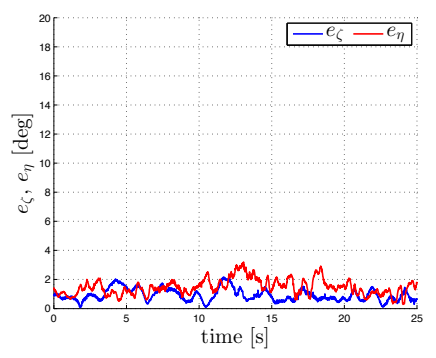

(a)

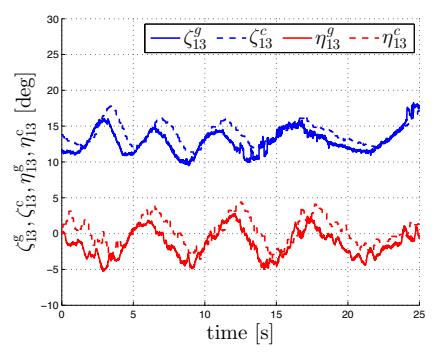

(b)
Fig. 9: Experiments: mean square measurement error (a) and measurement lag (b) when comparing the measures obtained from the on-board cameras with the one computed from the ground-truth.

\section{A. Environmental Scanning in case of Limited Field-of-View}

To overcome the restrictions due to the limited FOV in the horizontal plane, we forced every UAV to rotate with some desired rotation speed $\tilde{\omega}_{i, 3}(t)$ in order to 'scan' the environment and periodically detect all the other UAVs. This is recast into the problem of letting the UAVs tracking a time-varying desired bearing trajectory without affecting the geometrical shape of the formation, as explained in Sec. IV-D.

Every agent $i \in(1, \ldots, N)$ maintains a local estimation of 
the azimuth $\zeta_{i j}$ relative to another agent $j$ by means of the following dynamical system

$$
\dot{\xi}_{i j}=K_{\xi}\left(\zeta_{i j}-\xi_{i j}\right)-\dot{\psi}_{\mathcal{A}_{i}}
$$

where $\dot{\psi}_{\mathcal{A}_{i}}$ is the measured yaw-rate of the UAV, the estimation gain $K_{\xi}>0$ if a measurements $\zeta_{i j}$ is available, and $K_{\xi}=0$ otherwise. This simple estimation scheme will converge if the bearing-formation is kept close enough to the desired one, so that no additional dynamics influence the evolution of $\zeta_{i j}$. For instance, this estimation will not exactly track the real $\zeta_{i j}$ whenever a rotation command $w$ is applied with a wrong guess for $\hat{\delta}_{12}$.

Then we can freely design the scan rotation speed $\tilde{\omega}_{i, 3}(t)$ in (33-35) as long as we can guarantee that the relative bearing measurements are acquired often enough to refresh the estimation (51). We chose the following refreshing strategy: at the beginning of the task, every UAV performs a complete $360 \mathrm{deg}$ scan to initialize the estimations of all the needed relative azimuths. During normal motion, on the other hand, the $i$-th UAV will rotate towards the UAV that was not seen for the longest time, say $j$. The sign of $\tilde{\omega}_{i, 3}(t)$ is chosen in order to travel the smallest angle. When $j$ will be in the FOV of $i$, the measurement $\zeta_{i j}$ is plugged into (51), and the procedure is repeated for another agent $k$. Depending on the configuration of the formation, this strategy will make some agent to periodically invert the rotation direction, and others to persistently rotate in the same direction. Finally, if a UAV can measure the bearings relative to all the other UAVs, it will simply rotate to keep them in the most centered way.

We first tested this strategy in simulation assuming a horizontal FOV of $[-44,+44]$ deg. Figure 10 shows the results of 7 simulated quadrotors which, starting from the desired bearing formation, are commanded with translations and expansion rates while implementing the estimation law (51) with the proposed scanning strategy. Figure 10(a) reports the average quadratic error of the bearing formation computed from the ground-truth position measurements available in simulation. Note how this error is almost zero during the whole simulation, showing that the desired bearing-formation was indeed correctly realized. Figure 10(b) depicts the time behavior of an estimated azimuth (red line) and of the corresponding real value (blue line). One can appreciate how the estimation is able to track the true azimuth value also when the other UAV is out of the FOV (above the horizontal black line in the plot). In this case, the maximum estimation error was about $2 \mathrm{deg}$ and occurred when the other UAV was not in visibility.

We then repeated the same scenario in an experiment with 3 real quadrotors as before. All the bearing measurements were obtained from the cameras shown in Fig. 5(b), which have an horizontal FOV of about $[-44,+44]$ deg. Figure 11(a) shows again the mean square error of the bearing-formation from the ground-truth data. The error remains very small although the quadrotors are often using the estimated bearings from (51). Similarly to before, Fig. 11(b) reports the evolution of an estimated azimuth and the corresponding ground-truth value obtained from the external tracking system. In this case, the estimation degrades when the tracked UAV is outside of the camera FOV (above the horizontal black line in the plot), with

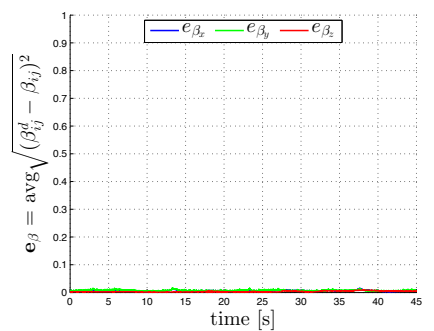

(a)

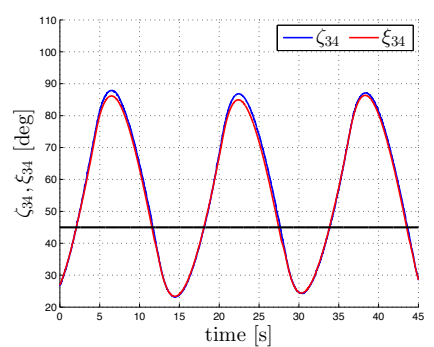

(b)
Fig. 10: Simulation with 7 quadrotors waving to overcome the limited FOV. (a): bearing formation error. (b): Estimated azimuth vs. groundtruth measure

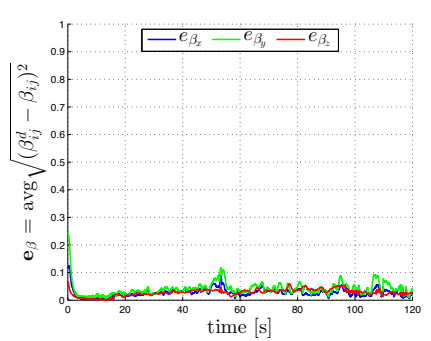

(a)

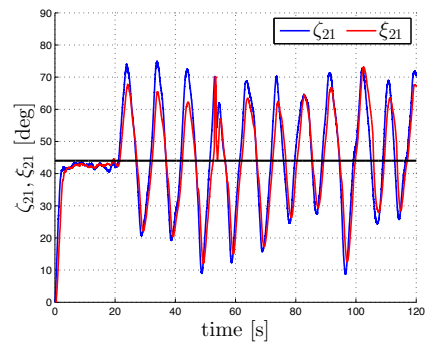

(b)
Fig. 11: Experiment with 3 quadrotors waving to overcome the limited FOV. (a): bearing formation error. (b) Estimated azimuth vs. ground-truth measure.

a maximum error of about $10 \mathrm{deg}$. This poorer performance w.r.t. the simulation case is due to all the unmodeled effects and real-world conditions and also to the wrong estimate $\hat{\delta}_{12}$ when commanding a rotation. However, we found the proposed controller was still able to keep the desired formation despite of these degrading effects.

Finally, we encourage the reader to watch the 4 videos (Extensions 1-4) associated with the paper where all the simulations and experiments discussed in this Section can be fully appreciated.

\section{CONClusion AND Future Work}

We presented a control framework for a group of UAVs bound to keep a bearing-formation and allowing for high-level group steering. We formally defined the concept of 3D bearingformations, and thoroughly studied the associated geometrical properties. We then devised a suitable formation controller based on only relative bearing measurements, and left the possibility for any high-level planner to control the remaining dofs of the UAV group. In the particular case of a human cooperator in charge of steering the formation, this was achieved by employing two force-feedback devices in order to provide haptic cues informative of group performances w.r.t. the human commands. The theoretical claims of the paper were finally validated by means of several HHIL simulations and experiments with 3 quadrotor UAVs where the needed bearing measurements were obtained from onboard cameras.

We are currently evaluating the possibility to estimate the actual inter-agent distances by exploiting the steadystate error present during synchronized rotations and due to 


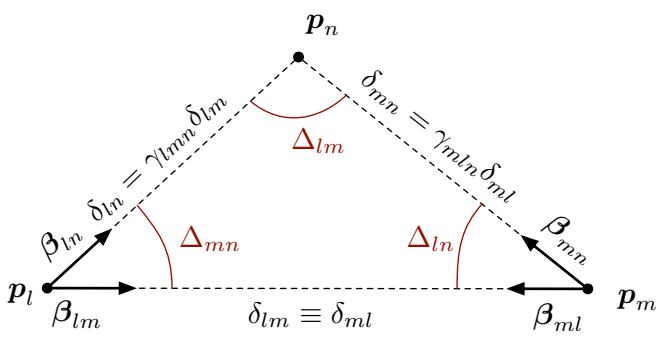

Fig. 12: The plane spanned by $\boldsymbol{p}_{l}, \boldsymbol{p}_{n}, \boldsymbol{p}_{m}$ and Property 2 .

wrong initial guesses in the quantity $\delta_{12}$. Estimating interdistances, or in general metric quantities, could also facilitate avoidance of obstacle collisions, an extension we are currently considering. Finally, in order to become fully independent from external tracking devices, we plan to perform some additional experiments incorporating the already developed visual-based techniques using onboard optical flow extraction and decomposition.

\section{ACKNOWLEDGEMENTS}

This research was partly supported by WCU (World Class University) program funded by the Ministry of Education, Science and Technology through the National Research Foundation of Korea (R31-10008).

ApPendix A: Index to Multimedia Extensions

The multimedia extensions are at: http://www.ijrr.org.

\begin{tabular}{l|l|l}
\hline Extension & Type & Description \\
\hline 1 & Video & HHIL first simulation \\
2 & Video & HHIL first experiment \\
3 & Video & HHIL simulation with limited FOV \\
4 & Video & HHIL experiment with limited FOV \\
\hline
\end{tabular}

\section{APPENDIX B: PROOFS}

Proof of Property 1. Multiplying both sides of (3) with $R_{i}$, we obtain $R_{i} \boldsymbol{\beta}_{i j}=\boldsymbol{p}_{i j} / \delta_{i j}$, and symmetrically $R_{j} \boldsymbol{\beta}_{j i}=$ $\boldsymbol{p}_{j i} / \delta_{j i}$. Noting that $\boldsymbol{p}_{i j}=-\boldsymbol{p}_{j i}$ and $\delta_{i j}=\delta_{j i}$, we can equate the left sides of the previous equations obtaining $R_{i} \boldsymbol{\beta}_{i j}=-R_{j} \boldsymbol{\beta}_{j i}$. This can be rewritten as ${ }^{j} R_{i} \boldsymbol{\beta}_{i j}=-\boldsymbol{\beta}_{j i}$ showing that ${ }^{j} R_{i}$ is the rotation matrix among the directions $\boldsymbol{\beta}_{i j}$ and $-\boldsymbol{\beta}_{j i}$. This consists of a rotation about the axis $\boldsymbol{v}_{i j}$ of an angle $\arccos \left(c_{i j}\right)$, and takes the exponential form (6). To conclude the proof, note that, in our case, ${ }^{i} R_{j}$ is always a rotation matrix of the form $R_{z}(\cdot)$ by construction. Therefore, for any $a=b= \pm(001)^{T}$, the equation ${ }^{i} R_{j} a=b$ does not admit a unique solution (any rotation matrix $R_{z}(\cdot)$ would be a solution), implying that ${ }^{i} R_{j}$ cannot be evaluated whenever $\boldsymbol{\beta}_{i j}= \pm\left(\begin{array}{lll}0 & 0 & 1\end{array}\right)^{T}$.

Finally, the last part is obtained by applying the first part of Property 1 twice.

Proof of Property 2. Figure 12 represents the plane spanned by the points $\boldsymbol{p}_{l}, \boldsymbol{p}_{m}, \boldsymbol{p}_{n}$. From the law of sines, it follows $\frac{\delta_{l n}}{\delta_{l m}}=\frac{\sin \left(\Delta_{l n}\right)}{\sin \left(\Delta_{l m}\right)}$, where $\sin \left(\Delta_{l n}\right)=\left\|\boldsymbol{\beta}_{m n} \times \boldsymbol{\beta}_{m l}\right\|$ and

$$
\begin{aligned}
\sin \left(\Delta_{l m}\right) & =\left\|\boldsymbol{\beta}_{n l} \times \boldsymbol{\beta}_{n m}\right\|=\left\|{ }^{l} R_{n} \boldsymbol{\beta}_{n l} \times{ }^{l} R_{n} \boldsymbol{\beta}_{n m}\right\|= \\
& =\left\|-\boldsymbol{\beta}_{l n} \times{ }^{l} R_{m}{ }^{m} R_{n} \boldsymbol{\beta}_{n m}\right\|,
\end{aligned}
$$

thus proving (9). Finally, (10) follows from (8) via suitable relabeling.

Proof of Property 3. Identity (14) follows from

$$
\begin{aligned}
\boldsymbol{\beta}_{m n} & =\frac{{ }^{m} R\left(\boldsymbol{p}_{n}-\boldsymbol{p}_{m}\right)}{\left\|\boldsymbol{p}_{n}-\boldsymbol{p}_{m}\right\|}= \\
& =\frac{{ }^{m} R\left(\boldsymbol{p}_{l}+R_{l} \boldsymbol{\beta}_{l n} \gamma_{l m n} \delta_{l m}-\boldsymbol{p}_{l}-R_{l} \boldsymbol{\beta}_{l m} \delta_{l m}\right)}{\left\|\boldsymbol{p}_{n}-\boldsymbol{p}_{m}\right\|}= \\
& =\frac{{ }^{m} R R_{l}\left(\boldsymbol{\beta}_{l n} \gamma_{l m n}-\boldsymbol{\beta}_{l m}\right)}{\left(\left\|\boldsymbol{p}_{n}-\boldsymbol{p}_{m}\right\| \delta_{l m}^{-1}\right)},
\end{aligned}
$$

where the denominator $\left\|\boldsymbol{p}_{n}-\boldsymbol{p}_{m}\right\| \delta_{l m}^{-1}=\left\|\boldsymbol{\beta}_{l n} \gamma_{l m n}-\boldsymbol{\beta}_{l m}\right\|$ since $\boldsymbol{\beta}_{m n}$ is a unit vector.

Proof of Lemma 1. Consider any realization $\left(\mathcal{G}, \boldsymbol{q}^{\alpha}\right)$. In order to prove the Lemma, we will show that, w.l.o.g., if $\boldsymbol{q}_{1}^{\alpha}, \delta_{12}\left(\boldsymbol{q}^{\alpha}\right)$ and all the relative bearings $\boldsymbol{\beta}\left(\boldsymbol{q}^{\alpha}\right)$ are known, then all the rotation matrices $R_{j}\left(\boldsymbol{q}^{\alpha}\right)$ and positions $\boldsymbol{p}_{j}^{\alpha}, j \neq 1$, can be explicitly obtained. In the following we omit the dependency from $\boldsymbol{q}^{\alpha}$ for conciseness.

First of all we note that $R_{j}=R_{1}{ }^{1} R_{j}$ and $\boldsymbol{p}_{j}^{\alpha}=\boldsymbol{p}_{1}^{\alpha}+$ $R_{1} \boldsymbol{\beta}_{1 j} \delta_{1 j}$, so that we can reduce the problem to the computation of ${ }^{1} R_{j} \forall j>1$ and $\delta_{1 j} \forall j>2$. If $\boldsymbol{\beta}_{1 j} \neq \pm\left(\begin{array}{lll}0 & 0 & 1\end{array}\right)^{T}$ then ${ }^{1} R_{j}$ follows from (6) using the bearings $\boldsymbol{\beta}_{1 j}$ and $\boldsymbol{\beta}_{j 1}$.

If $\boldsymbol{\beta}_{1 j}= \pm\left(\begin{array}{lll}0 & 0 & 1\end{array}\right)^{T}$ (i.e., agents 1 and $j$ are on on top of each other), let $h$ be any agent such that $\boldsymbol{\beta}_{1 h} \neq \pm\left(\begin{array}{lll}0 & 0 & 1\end{array}\right)^{T}$ (nondegeneracy guarantees existence of at least one such agent). Then, one can compute ${ }^{1} R_{h}$ and ${ }^{h} R_{j}$ from $\boldsymbol{\beta}_{1 h}, \boldsymbol{\beta}_{h 1}, \boldsymbol{\beta}_{h j}, \boldsymbol{\beta}_{j h}$ using (6) twice, to finally obtain ${ }^{1} R_{j}={ }^{1} R_{h}{ }^{h} R_{j}$.

As for the inter-distances, since $\boldsymbol{q}^{\alpha}$ is non-degenerate, there exists at least an agent $h$ such that $\boldsymbol{\beta}_{12} \neq \pm \boldsymbol{\beta}_{1 h}$. Then, applying (8) with $(l, m, n)=(1,2, h)$, one can obtain $\delta_{1 h}=\gamma_{12 h} \delta_{12}$ and $\delta_{2 h}=\gamma_{21 h} \delta_{12}$ as a function of $\delta_{12}$ and the needed relative bearings. Finally, consider the case $j \neq 1,2, h$ : if $\boldsymbol{\beta}_{1 j} \neq \pm \boldsymbol{\beta}_{12}$ then $\delta_{1 j}=\gamma_{12 j} \delta_{12}$, otherwise $\delta_{1 j}=\gamma_{1 h j} \delta_{1 h}$, thus concluding the proof.

Proof of Lemma 2. It is easy to check that non-degeneracy implies the existence of at least one relabeling meeting the requirement $\boldsymbol{\beta}_{12}\left(\boldsymbol{q}^{\alpha}\right) \neq \pm \boldsymbol{\beta}_{13}\left(\boldsymbol{q}^{\alpha}\right) \neq \pm\left(\begin{array}{lll}0 & 0 & 1\end{array}\right)^{T}$. Furthermore, the equivalence follows from the fact that $\left(\hat{\mathcal{G}}, \boldsymbol{\beta}_{\hat{\mathcal{E}}}^{\alpha}\right)$ has been generated from a realization of $(\mathcal{G}, \boldsymbol{\alpha})$. The linearity can be checked computing the cardinality of $\hat{\mathcal{E}}$ that is $|\hat{\mathcal{E}}|=2(N-$ 1) $+\left|\mathcal{I}_{2}\right|+\left|\mathcal{I}_{3}\right|=3 N-4$, which is linear in the number of agents $N$.

In order to prove rigidity we show that all the bearings $\boldsymbol{\beta}_{i j}\left(\boldsymbol{q}^{\alpha}\right)$ with $(i, j) \notin \hat{\mathcal{E}}$ can be uniquely computed from the bearings in $\boldsymbol{\beta}_{\hat{\mathcal{E}}}^{\alpha}$. We omit the dependency from $\boldsymbol{q}^{\alpha}$ for brevity. All the bearings $\boldsymbol{\beta}_{2 j}, \forall j \in \mathcal{I}_{2}$, can be computed by evaluating $\gamma_{1 j 2}$ from (8) with $(l, m, n)=(1, j, 2)$, and then applying (14) with $(l, m, n)=(1,2, j)$ and noting that $\gamma_{12 j}=\gamma_{1 j 2}^{-1}$. Similarly we can determine $\boldsymbol{\beta}_{3 j} \forall j \in \mathcal{I}_{3}$.

The rotation matrixes of the form ${ }^{j} R_{1}, \forall j>1$, can be computed as follows: if $\boldsymbol{\beta}_{1 j} \neq \pm(001)$ then ${ }^{j} R_{1}$ is determined using (6) with $(i, j)=(1, j)$. Otherwise, if $j \in \mathcal{I}_{2}$, then ${ }^{j} R_{1}$ can be evaluated as ${ }^{j} R_{2}^{2} R_{1}$ using (6) twice, first with $(i, j)=(j, 2)$ and then with $(i, j)=(2,1)$.

Finally, if $j \in \mathcal{I}_{3}$, we can determine ${ }^{j} R_{1}={ }^{j} R_{3}{ }^{3} R_{1}$ in a similar fashion. This further allows to obtain any ${ }^{j} R_{i}, \forall j \neq i$, 
since ${ }^{j} R_{i}={ }^{j} R_{1}{ }^{i} R_{1}^{T}$. Consequently, for any known relative bearing $\boldsymbol{\beta}_{i j}$, we also get $\boldsymbol{\beta}_{j i}=-{ }^{j} R_{i} \boldsymbol{\beta}_{i j}$.

To conclude this part of the proof, we show how to compute $\boldsymbol{\beta}_{i j}$ for any $i>3, j>i$. To this end it is sufficient to apply (14) using $(l, m, n)=(1, i, j)$, where $\gamma_{1 j i}$ can be obtained as $\gamma_{12 i} / \gamma_{12 j}$ if $j \in \mathcal{I}_{2}$, or as $\gamma_{13 i} / \gamma_{13 j}$ if $j \in \mathcal{I}_{3}$.

Finally, in order to prove minimality, we show that rigidity is lost if any relative bearing is removed from $\hat{\mathcal{E}}$. First, if any bearing of the form $\boldsymbol{\beta}_{1 i}$ or $\boldsymbol{\beta}_{i 1}, i>1$, is removed, then ${ }^{i} R_{1}$ can be any rotation matrix of the form $R_{z}(\cdot)$, thus contradicting Lemma 1. Second, if any bearing of the form $\boldsymbol{\beta}_{j i}$, with $i=$ $2,3, j \in \mathcal{I}_{i}$, is removed, then the ratio $\gamma_{1 i j}$ can take any value, i.e., $\delta_{1 i}$ and $\delta_{1 j}$ can be chosen freely, thus leading again to a contradiction with Lemma 1 . Therefore no relative bearing can be removed from $\hat{\mathcal{E}}$ without losing rigidity, hence proving the last part of the statement.

Proof of Lemma 3. The time derivative of a bearing $\dot{\boldsymbol{\beta}}_{i j}$ is obtained by differentiating (3) as

$$
\dot{\boldsymbol{\beta}}_{i j}=\frac{1}{\delta_{i j}} R\left[\dot{\psi}_{i} S \hat{\boldsymbol{p}}_{i j}+P\left(\hat{\boldsymbol{p}}_{i j}\right) \dot{\boldsymbol{p}}_{i j}\right],
$$

where $P\left(\hat{\boldsymbol{p}}_{i j}\right)=\left(I-\hat{\boldsymbol{p}}_{i j} \hat{\boldsymbol{p}}_{i j}^{T}\right) \in \mathbb{R}^{3 \times 3}$ is the projection matrix onto the plane perpendicular to $\hat{\boldsymbol{p}}_{i j}$. Imposing $\dot{\boldsymbol{\beta}}_{i j}=0$ in (52) results in the following condition

$$
0=\dot{\psi}_{i} S \boldsymbol{p}_{i j}+P\left(\hat{\boldsymbol{p}}_{i j}\right) \dot{\boldsymbol{p}}_{i j},
$$

which, combined with the symmetric condition $\dot{\boldsymbol{\beta}}_{j i}=0$, yields

$$
\left(\dot{\psi}_{j}-\dot{\psi}_{i}\right) S \boldsymbol{p}_{i j}=\dot{\psi}_{i j} S \boldsymbol{p}_{i j}=0
$$

where we exploited the fact that $\boldsymbol{p}_{i j}=-\boldsymbol{p}_{j i}, \dot{\boldsymbol{p}}_{i j}=-\dot{\boldsymbol{p}}_{j i}$, $\hat{\boldsymbol{p}}_{i j}=-\hat{\boldsymbol{p}}_{j i}$, and $P\left(\hat{\boldsymbol{p}}_{j i}\right)=P\left(\hat{\boldsymbol{p}}_{i j}\right)$.

In the particular case of $N=3$ agents considered here, the constraints (54) for all possible agent pairs are simply

$$
\dot{\psi}_{12} S \boldsymbol{p}_{12}=0, \quad \dot{\psi}_{13} S \boldsymbol{p}_{13}=0, \quad \dot{\psi}_{23} S \boldsymbol{p}_{23}=0 .
$$

Being ker $S=\operatorname{span}\left\{\left(\begin{array}{lll}0 & 0 & 1\end{array}\right)^{T}\right\}$, these can be satisfied when either $\boldsymbol{p}_{i j} \propto(001)^{T}$ or $\dot{\psi}_{i j}=0$. However, since the positions $\boldsymbol{p}_{1}, \boldsymbol{p}_{2}$, and $\boldsymbol{p}_{3}$ are not aligned, there exist at least two vectors among $\left(\boldsymbol{p}_{12}, \boldsymbol{p}_{13}, \boldsymbol{p}_{23}\right)$ not in ker $S$. Therefore, we can conclude that at least two entries among $\left(\dot{\psi}_{13}, \dot{\psi}_{12}, \dot{\psi}_{23}\right)$ must be zero. Being $\dot{\psi}_{13}=\dot{\psi}_{12}+\dot{\psi}_{23}$ by construction, it necessarily follows that $\dot{\psi}_{13}=\dot{\psi}_{12}=\dot{\psi}_{23}=0$.

Consider now the (globally invertible) change of coordinates

$$
\Pi \boldsymbol{q}=\boldsymbol{q}^{\prime}=\left(\boldsymbol{p}_{1} \boldsymbol{p}_{12} \boldsymbol{p}_{13} \psi_{1} \psi_{12} \psi_{13}\right)^{T}
$$

and the associated new generalized velocities

$$
\Pi \dot{\boldsymbol{q}}=\dot{\boldsymbol{q}}^{\prime}=\left(\dot{\boldsymbol{p}}_{1} \dot{\boldsymbol{p}}_{12} \dot{\boldsymbol{p}}_{13} \dot{\psi}_{1} \dot{\psi}_{12} \dot{\psi}_{13}\right)^{T},
$$

with $\Pi \in \mathbb{R}^{12 \times 12}$, the determinant $\operatorname{det} \Pi \neq 0$, being the nonsingular transformation matrix associated to this change of coordinates. Constraints (53) can be rewritten in a matrix form in terms of $\boldsymbol{q}^{\prime}$ and $\dot{\boldsymbol{q}}^{\prime}$ as:

$\underbrace{\left[\begin{array}{cccccc}\mathbf{0}_{3 \times 3} & P\left(\hat{\boldsymbol{p}}_{12}\right) & \mathbf{0}_{3 \times 3} & S \boldsymbol{p}_{12} & \mathbf{0}_{3} & \mathbf{0}_{3} \\ \mathbf{0}_{3 \times 3} & \mathbf{0}_{3 \times 3} & P\left(\hat{\boldsymbol{p}}_{13}\right) & S \boldsymbol{p}_{13} & \mathbf{0}_{3} & \mathbf{0}_{3} \\ \mathbf{0}_{3 \times 3} & -P\left(\hat{\boldsymbol{p}}_{23}\right) & P\left(\hat{\boldsymbol{p}}_{23}\right) & S \boldsymbol{p}_{23} & \mathbf{0}_{3} & \mathbf{0}_{3} \\ \mathbf{0}_{3}^{T} & \mathbf{0}_{3}^{T} & \mathbf{0}_{3}^{T} & \mathbf{0}_{3}^{T} & 1 & 0 \\ \mathbf{0}_{3}^{T} & \mathbf{0}_{3}^{T} & \mathbf{0}_{3}^{T} & \mathbf{0}_{3}^{T} & 0 & 1\end{array}\right]}_{A\left(\boldsymbol{q}^{\prime}\right) \in \mathbb{R}^{11 \times 12}} \dot{\boldsymbol{q}}^{\prime}=A\left(\boldsymbol{q}^{\prime}\right) \dot{\boldsymbol{q}}^{\prime}=0$,

where $0_{3 \times 3}$ is the $3 \times 3$ null matrix, and the facts that $\dot{\boldsymbol{p}}_{23}=$ $\dot{\boldsymbol{p}}_{13}-\dot{\boldsymbol{p}}_{12}$ and $\dot{\psi}_{12}=\dot{\psi}_{2}-\dot{\psi}_{1}=0$ were exploited.

We can then identify $T_{\boldsymbol{q}^{\prime}} \mathcal{F}_{\mathcal{K}}(\boldsymbol{q})$ with $\operatorname{ker} A\left(\boldsymbol{q}^{\prime}\right)$. Note that, although $\max \left(\operatorname{rank}\left(A\left(\boldsymbol{q}^{\prime}\right)\right)\right)=11$, implying that $\min \left(\operatorname{dim} \operatorname{ker} A\left(\boldsymbol{q}^{\prime}\right)\right)=1$, we already know that $\operatorname{dim} \operatorname{ker} A\left(\boldsymbol{q}^{\prime}\right)=5$ (resp. $\operatorname{rank}\left(A\left(\boldsymbol{q}^{\prime}\right)\right)=7$ ) by construction. In fact, being $\mathcal{F}_{\mathcal{K}}(\boldsymbol{q})$ a regular manifold of dimension 5 (Lemma 1), the dimension of its tangent space at any point, i.e., of $\operatorname{ker}\left(A\left(\boldsymbol{q}^{\prime}\right)\right)$, must be necessarily 5. An explicit expression for $T_{\boldsymbol{q}^{\prime}} \mathcal{F}_{\mathcal{K}}(\boldsymbol{q})$ can then be found by inspection as $T_{\boldsymbol{q}^{\prime}} \mathcal{F}_{\mathcal{K}}(\boldsymbol{q})=\operatorname{ker} A\left(\boldsymbol{q}^{\prime}\right)=\mathcal{R}\left(T_{3}^{\prime}\right)$ where

$T_{3}^{\prime}=\left[\begin{array}{cccccc}I_{3} & \mathbf{0}_{3 \times 3} & \mathbf{0}_{3 \times 3} & \mathbf{0}_{3} & \mathbf{0}_{3} & \mathbf{0}_{3} \\ \mathbf{0}_{3}^{T} & \hat{\boldsymbol{p}}_{12}^{T} & \gamma_{123} \hat{\boldsymbol{p}}_{13}^{T} & 0 & 0 & 0 \\ \mathbf{0}_{3}^{T} & -\left(S \boldsymbol{p}_{12}\right)^{T} & -\left(\boldsymbol{S}_{13}\right)^{T} & 1 & 0 & 0\end{array}\right]^{T} \in \mathbb{R}^{12 \times 5}$.

In fact, letting $\boldsymbol{t}_{i}^{\prime} \in \mathbb{R}^{12}$ be the $i$-th column of $T_{3}^{\prime}$, on can easily check that $\left(\boldsymbol{t}_{1}^{\prime T}, \boldsymbol{t}_{2}^{\prime T}, \boldsymbol{t}_{3}^{\prime T}\right)^{T}$ are in $\operatorname{ker} A\left(\boldsymbol{q}^{\prime}\right)$. Furthermore, $\boldsymbol{t}_{4}^{\prime} \in \operatorname{ker} A\left(\boldsymbol{q}^{\prime}\right)$ since $P\left(\hat{\boldsymbol{p}}_{12}\right) \hat{\boldsymbol{p}}_{12}=\mathbf{0}_{3}$ and $P\left(\hat{\boldsymbol{p}}_{13}\right) \hat{\boldsymbol{p}}_{13}=\mathbf{0}_{3}$ by construction, and $-P\left(\hat{\boldsymbol{p}}_{23}\right) \hat{\boldsymbol{p}}_{12}+\gamma_{123} P\left(\hat{\boldsymbol{p}}_{23}\right) \hat{\boldsymbol{p}}_{13}$ can be rewritten as

$$
\begin{aligned}
& \frac{1}{\delta_{12}}\left(-P\left(\hat{\boldsymbol{p}}_{23}\right) \boldsymbol{p}_{12}+P\left(\hat{\boldsymbol{p}}_{23}\right) \boldsymbol{p}_{13}\right)= \\
& \frac{1}{\delta_{12}}\left(-P\left(\hat{\boldsymbol{p}}_{23}\right)\left(\boldsymbol{p}_{13}-\boldsymbol{p}_{23}\right)+P\left(\hat{\boldsymbol{p}}_{23}\right) \boldsymbol{p}_{13}\right)= \\
& \frac{1}{\delta_{12}}\left(-P\left(\hat{\boldsymbol{p}}_{23}\right) \boldsymbol{p}_{13}+P\left(\hat{\boldsymbol{p}}_{23}\right) \boldsymbol{p}_{13}\right)=\mathbf{0}_{3} .
\end{aligned}
$$

Finally, $\boldsymbol{t}_{5}^{\prime} \in \operatorname{ker} A\left(\boldsymbol{q}^{\prime}\right)$ since $-P\left(\hat{\boldsymbol{p}}_{12}\right) S \boldsymbol{p}_{12}+S \boldsymbol{p}_{12}=$ $-S \boldsymbol{p}_{12}+S \boldsymbol{p}_{12}=\mathbf{0}_{3}$ and, similarly, $-P\left(\hat{\boldsymbol{p}}_{13}\right) S \boldsymbol{p}_{13}+$ $S \boldsymbol{p}_{13}=-S \boldsymbol{p}_{13}+S \boldsymbol{p}_{13}=\mathbf{0}_{3}$, implying that $P\left(\hat{\boldsymbol{p}}_{23}\right) S \boldsymbol{p}_{12}$ $P\left(\hat{\boldsymbol{p}}_{23}\right) S \boldsymbol{p}_{13}+S \boldsymbol{p}_{23}=P\left(\hat{\boldsymbol{p}}_{23}\right) S \boldsymbol{p}_{13}-P\left(\hat{\boldsymbol{p}}_{23}\right) S \boldsymbol{p}_{23}-$ $P\left(\hat{\boldsymbol{p}}_{23}\right) S \boldsymbol{p}_{13}+S \boldsymbol{p}_{23}=-S \boldsymbol{p}_{23}+S \boldsymbol{p}_{23}=\mathbf{0}_{3}$.

Note that $\operatorname{rank}\left(T_{3}^{\prime}\right)=5$ as expected. This can be easily verified by noting the 'upper triangular' form of $T_{3}^{\prime}$. As a last step, we can recover the sought $T_{3}$ in (21) by going back to the original coordinates $\boldsymbol{q}$, i.e., by taking $T_{3}=\Pi^{-1} T_{3}^{\prime}$. The resulting matrix is explicitly shown in (21).

\section{REFERENCES}

[1] A. Howard, L. E. Parker, and G. S. Sukhatme, "Experiments with a large heterogeneous mobile robot team: Exploration, mapping, deployment and detection," International Journal of Robotics Research, vol. 25, no. 5-6, pp. 431-447, 2006.

[2] A. Franchi, L. Freda, G. Oriolo, and M. Vendittelli, "The sensor-based random graph method for cooperative robot exploration," IEEE/ASME Trans. on Mechatronics, vol. 14, no. 2, pp. 163-175, 2009.

[3] M. Schwager, B. Julian, M. Angermann, and D. Rus, "Eyes in the sky: Decentralized control for the deployment of robotic camera networks," Proceedings of the IEEE, vol. 99, no. 9, pp. 1541-1561, 2011.

[4] A. Renzaglia, L. Doitsidis, A. Martinelli, and E. B. Kosmatopoulos, "Multi-robot three dimensional coverage of unknown areas," International Journal of Robotics Research, vol. 31, no. 6, pp. 738-752, 2012.

[5] sFly, "EU Collaborative Project ICT-231855," http://www.sfly.org/.

[6] AIRobots, "EU Collaborative Project ICT-248669," www.airobots.eu.

[7] ARCAS, "EU Collaborative Project ICT-287617," www.arcas-project. eu.

[8] A. Franchi, P. Stegagno, and G. Oriolo, "Probabilistic mutual localization in multi-agent systems from anonymous position measures," in 49th IEEE Conf. on Decision and Control, Atlanta, GA, Dec. 2010, pp. 6534-6540

[9] J. W. Durham, A. Franchi, and F. Bullo, "Distributed pursuit-evasion without global localization via local frontiers," Autonomous Robots, vol. 32, no. 1, pp. 81-95, 2012. 
[10] M. Cognetti, P. Stegagno, A. Franchi, G. Oriolo, and H. H. Bülthoff, "3D mutual localization with anonymous bearing measurements," in 2012 IEEE Int. Conf. on Robotics and Automation, St. Paul, MN, May 2012, pp. 791-798.

[11] R. Murphy, S. Tadokoro, D. Nardi, A. Jacoff, P. Fiorini, H. Choset, and A. Erkmen, "Search and rescue robotics," in Springer Handbook of Robotics, B. Siciliano and O. Khatib, Eds. Springer, 2008, pp. 11511173.

[12] P. F. Hokayem and M. W. Spong, "Bilateral teleoperation: An historical survey," Automatica, vol. 42, no. 12, pp. 2035-2057, 2006.

[13] T. M. Lam, H. W. Boschloo, M. Mulder, and M. M. V. Paassen, "Artificial force field for haptic feedback in UAV teleoperation," IEEE Trans. on Systems, Man, \& Cybernetics. Part A: Systems \& Humans, vol. 39, no. 6, pp. 1316-1330, 2009.

[14] D. A. Abbink, M. Mulder, F. C. T. van der Helm, M. Mulder, and E. R. Boer, "Measuring neuromuscular control dynamics during car following with continuous haptic feedback," IEEE Trans. on Systems, Man, \& Cybernetics. Part B: Cybernetics, vol. 41, no. 5, pp. 1239-1249, 2011.

[15] A. K. Das, R. Fierro, V. Kumar, P. Ostrowski, J. Spletzer, and C. J. Taylor, "A vision-based formation control framework," IEEE Trans. on Robotics and Automation, vol. 18, no. 5, pp. 813-825, 2002.

[16] R. V. O. Shakernia and S. Sastry, "Formation control of nonholonomic mobile robots with omnidirectional visual servoing and motion segmentation," in 2003 IEEE Int. Conf. on Robotics and Automation, Taipei, Taiwan, Sep. 2003, pp. 584-589.

[17] E. N. Johnson, A. J. Calise, R. Sattigeri, Y. Watanabe, and V. Madyastha, "Approaches to vision-based formation control," in 43th IEEE Conf. on Decision and Control, Paradise Island, Bahamas, Jan. 2004, pp. 16431648.

[18] O. A. A. Orqueda and R. Fierro, "Robust vision-based nonlinear formation control," in 2006 American Control Conference, Minneapolis, MN, Jun. 2006, pp. 1422-1427.

[19] N. Moshtagh, N. Michael, A. Jadbabaie, and K. Daniilidis, "Visionbased, distributed control laws for motion coordination of nonholonomic robots," IEEE Trans. on Robotics, vol. 25, no. 4, pp. 851-860, 2009.

[20] G. L. Mariottini, F. Morbidi, D. Prattichizzo, N. Vander Valk, N. Michael, G. Pappas, and K. Daniilidis, "Vision-based localization for leader-follower formation control," IEEE Trans. on Robotics, vol. 25, no. 6, pp. 1431-1438, 2009.

[21] L. Kneip, A. Martinelli, S. Weiss, D. Scaramuzza, and R. Siegwart, "Closed-form solution for absolute scale velocity determination combining inertial measurements and a single feature correspondence," in 2011 IEEE Int. Conf. on Robotics and Automation, Shanghai, China, May 2011, pp. 4546-4553.

[22] J. Kelly and G. S. Sukhatme, "Visual-inertial sensor fusion: Localization, mapping and sensor-to-sensor self-calibration," International Journal of Robotics Research, vol. 30, no. 1, pp. 56-79, 2011.

[23] C. Belta and V. Kumar, "Abstraction and control for groups of robots," IEEE Trans. on Robotics, vol. 20, no. 5, pp. 865-875, 2004.

[24] C. A. Kitts and I. Mas, "Cluster space specification and control of mobile multirobot systems," IEEE/ASME Trans. on Mechatronics, vol. 14, no. 2, pp. 207-218, 2009.

[25] G. Antonelli, F. Arrichiello, and S. Chiaverini, "Experiments of formation control with multirobot systems using the null-space-based behavioral control," IEEE Trans. on Control Systems Technology, vol. 17, no. 5, pp. 1173-1182, 2009.

[26] D. Lee and M. W. Spong, "Bilateral teleoperation of multiple cooperative robots over delayed communication network: theory," in 2005 IEEE Int. Conf. on Robotics and Automation, Barcelona, Spain, Apr. 2005, pp. 360-365.

[27] E. J. Rodríguez-Seda, J. J. Troy, C. A. Erignac, P. Murray, D. M. Stipanović, and M. W. Spong, "Bilateral teleoperation of multiple mobile agents: Coordinated motion and collision avoidance," IEEE Trans. on Control Systems Technology, vol. 18, no. 4, pp. 984-992, 2010.

[28] D. Lee, A. Franchi, P. Robuffo Giordano, H. I. Son, and H. H. Bülthoff, "Haptic teleoperation of multiple unmanned aerial vehicles over the internet," in 2011 IEEE Int. Conf. on Robotics and Automation, Shanghai, China, May 2011, pp. 1341-1347.

[29] A. Franchi, P. Robuffo Giordano, C. Secchi, H. I. Son, and H. H. Bülthoff, "A passivity-based decentralized approach for the bilateral teleoperation of a group of UAVs with switching topology," in 2011 IEEE Int. Conf. on Robotics and Automation, Shanghai, China, May 2011, pp. 898-905.

[30] A. Franchi, C. Secchi, M. Ryll, H. H. Bülthoff, and P. Robuffo Giordano, "Shared control: Balancing autonomy and human assistance with a group of quadrotor UAVs." IEEE Robotics \& Automation Magazine, vol. 19, no. $3,2012$.
[31] A. Franchi, C. Secchi, H. I. Son, H. H. Bülthoff, and P. Robuffo Giordano, "Bilateral teleoperation of groups of mobile robots with time-varying topology," IEEE Trans. on Robotics, vol. In Press, Electronically published at http://ieeexplore.ieee.org/xpl/articleDetails.jsp?arnumber=6199993, 2013.

[32] C. Secchi, A. Franchi, H. H. Bülthoff, and P. Robuffo Giordano, "Bilateral teleoperation of a group of UAVs with communication delays and switching topology," in 2012 IEEE Int. Conf. on Robotics and Automation, St. Paul, MN, May 2012, pp. 4307-4314.

[33] P. Robuffo Giordano, A. Franchi, C. Secchi, and H. H. Bülthoff, "Passivity-based decentralized connectivity maintenance in the bilateral teleoperation of multiple UAVs," in 2011 Robotics: Science and Systems, Los Angeles, CA, Jun. 2011.

[34] - "Experiments of passivity-based bilateral aerial teleoperation of a group of UAVs with decentralized velocity synchronization," in 2011 IEEE/RSJ Int. Conf. on Intelligent Robots and Systems, San Francisco, CA, Sep. 2011, pp. 163-170.

[35] R. Mahony, T. Hamel, and J.-M. Pflimlin, "Nonlinear complementary filters on the special orthogonal group," IEEE Trans. on Automatic Control, vol. 53, no. 5, pp. 1203-1218, 2008.

[36] M. Fliess, J. Lévine, P. Martin, and P. Rouchon, "Flatness and defect of nonlinear systems: Introductory theory and examples," International Journal of Control, vol. 61, no. 6, pp. 1327-1361, 1995.

[37] A. Isidori, Nonlinear Control Systems, 3rd edition. Springer, 1995.

[38] M. J. V. Nieuwstadt and R. M. Murray, "Real-time trajectory generation for differentially flat systems," International Journal on Robust and Nonlinear Control, vol. 8, pp. 995-1020, 1998.

[39] V. Mistler, A. Benallegue, and N. K. M'Sirdi, "Exact linearization and noninteracting control of a 4 rotors helicopter via dynamic feedback," in 10th IEEE Int. Symp. on Robots and Human Interactive Communications, Bordeaux, Paris, France, Sep. 2001, pp. 586-593.

[40] T. Eren, W. Whiteley, A. S. Morse, P. N. Belhumeur, and B. D. O. Anderson, "Sensor and network topologies of formations with direction, bearing, and angle information between agents," in 42th IEEE Conf. on Decision and Control, Maui, HI, Dec. 2003, pp. 3064-3069.

[41] T. Eren, W. Whiteley, and P. Belhumeur, "Using angle of arrival (bearing) information in network localization," in 45th IEEE Conf. on Decision and Control, San Diego, CA, Jan. 2006, pp. 4676-4681.

[42] N. A. Lynch, Distributed Algorithms. Morgan Kaufmann, 1997.

[43] D. J. Lee and K. Huang, "Passive-set-position-modulation framework for interactive robotic systems," IEEE Trans. on Robotics, vol. 26, no. 2, pp. 354-369, 2010.

[44] N. Hogan, "Controlling impedance at the man/machine," in 1989 IEEE Int. Conf. on Robotics and Automation, Scottsdale, AZ, May 1989, pp. $1626-1631$.

[45] N. Michael, D. Mellinger, Q. Lindsey, and V. Kumar, "The GRASP multiple micro-UAV testbed," IEEE Robotics \& Automation Magazine, vol. 17 , no. 3 , pp. $56-65,2010$.

[46] J. Lächele, A. Franchi, H. H. Bülthoff, and P. Robuffo Giordano, "SwarmSimX: Real-time simulation environment for multi-robot systems," in 3rd Int. Conf. on Simulation, Modeling, and Programming for Autonomous Robots, Tsukuba, Japan, Nov. 2012.

[47] V. Grabe, H. H. Bülthoff, and P. Robuffo Giordano, "On-board velocity estimation and closed-loop control of a quadrotor UAV based on optical flow," in 2012 IEEE Int. Conf. on Robotics and Automation, St. Paul, MN, May 2012, pp. 491-497. 